Draft Version SEPtember 30, 2018

Preprint typeset using $\mathrm{L}_{\mathrm{A}}^{\mathrm{A}} \mathrm{E} \mathrm{X}$ style emulateapj v. 5/2/11

\title{
THE GIANT MOLECULAR CLOUD ENVIRONMENTS OF INFRARED DARK CLOUDS
}

\author{
Audra K. HERnANDEZ \\ Department of Astronomy, University of Wisconsin, 475 North Charter Street, Madison, WI 53706, USA; \\ hernande@astro.wisc.edu \\ JONATHAN C. TAN \\ Departments of Astronomy \& Physics, University of Florida, Gainesville, FL 32611, USA; \\ jt@astro.ufl.edu \\ Draft version September 30, 2018
}

\begin{abstract}
We study Giant Molecular Cloud (GMC) environments surrounding 10 Infrared Dark Clouds (IRDCs), using ${ }^{13} \mathrm{CO}(1-0)$ emission from the Galactic Ring Survey. We measure physical properties of these IRDCs/GMCs on a range of scales extending to radii, $R$, of $30 \mathrm{pc}$. By comparing different methods for defining cloud boundaries and for deriving mass surface densities and velocity dispersions, we settle on a preferred "CE, $\tau, \mathrm{G}$ " method of "Connected Extraction" in position-velocity space plus Gaussian fitting to opacity-corrected line profiles for velocity dispersion and mass estimation. We examine how cloud definition affects measurements of the magnitude and direction of line-of-sight velocity gradients and velocity dispersions, including associated dependencies on size scale. CE, $\tau$, Gdefined GMCs show velocity dispersion versus size relations $\sigma \propto s^{1 / 2}$, which are consistent with the large-scale gradients being caused by turbulence. However, IRDCs have velocity dispersions that are moderately enhanced above those predicted by this scaling relation. We examine the dynamical state of the clouds finding mean virial parameters $\bar{\alpha}_{\text {vir }} \simeq 1.0$ for GMCs and 1.6 for IRDCs, broadly consistent with models of magnetized virialized pressure-confined polytropic clouds, but potentially indicating that IRDCs have more disturbed kinematics. CE, $\tau$, G-defined clouds exhibit a tight correlation of $\sigma / R^{1 / 2} \propto \Sigma^{n}$, with $n \simeq 0.7$ for GMCs and 1.3 for IRDCs (c.f., a value of 0.5 expected for a population of virialized clouds). We conclude that while GMCs show evidence for virialization over a range of scales, IRDCs may be moderately super virial. Alternatively, IRDCs could be virialized but have systematically different ${ }^{13} \mathrm{CO}$ gas phase abundances, i.e., due to freeze-out, affecting mass estimations.
\end{abstract}

Subject headings: ISM: clouds - ISM: kinematics and dynamics - Stars: formation

\section{INTRODUCTION}

Galactic star formation resides mostly within Giant Molecular Clouds (GMCs), conventionally defined to have masses $\geq 10^{4} M_{\odot}$ and observed to extend up to several $\times 10^{6} M_{\odot}$ (e.g., Blitz 1993, Williams et al. 2000; McKee \& Ostriker 2007). With typical mass surface densities of $\sim 100 M_{\odot} \mathrm{pc}^{-2}$, GMCs have mean radial sizes of $\sim 6-100 \mathrm{pc}$, assuming simple circular symmetry. However, GMCs are highly irregular and hierarchical structures. Their dense clumps can spawn stellar clusters and associations, creating the bulk of the Galactic field star population. The efficiency and rate of star formation from these clumps is relatively low, i.e., a few percent per local free-fall time (Zuckerman \& Evans 1974, Krumholz \& Tan 2007). This appears to be mostly because much of the GMC material is stable with respect to gravitational collapse, especially material below a threshold $A_{V} \sim 10 \mathrm{mag}$ (e.g., Lada et al. 2010). Higher total star formation efficiencies, $\sim 10-50 \%$, appear to be possible in the star-forming clumps that form at least moderately bound clusters (Lada \& Lada 2003).

Different theoretical models of the processes that create star-forming clumps within GMCs or prevent overdensities in the bulk of the cloud are actively debated. These processes include the regulation of star formation and stabilization of gas by magnetic fields (McKee 1989, Mouschovias 2001) or turbulence (Krumholz \& McKee 2005, Padoan \& Nordlund 2011), and/or the initiation of star formation by discrete triggering events, such as converging atomic flows (e.g., Heitsch et al. 2006), cloud collisions (e.g., Tan 2000) or stellar feedback (e.g., Samal et al. 2014).

Infrared dark clouds (IRDCs) are likely to be examples of early stage star-forming clumps (e.g., Perault et al. 1996, Egan et al. 1998: Carey et al. 2000, Rathborne et al. 2006; Butler \& Tan 2009, Peretto \& Fuller 2009: Battersby et al. 2010). Thus their study may help us understand the processes that initiate star formation in GMCs. There have been many investigations of the internal properties of IRDCs, including their temperatures (e.g., Pillai et al. 2006: Peretto et al. 2010; Ragan et al. 2011; Chira et al. 2013), mass surface density structure (e.g., Butler \& Tan 2009, Peretto \& Fuller 2009: Ragan et al. 2011; Butler \& Tan 2012; Kainulainen \& Tan 2013; Butler et al. 2014), kinematics (e.g., Henshaw et al. 2013; Jiménez-Serra et al. 2014) and dynamics (e.g., Hernandez \& Tan 2011 Hernandez et al. 2012), CO depletion (Fontani et al. 2006; Hernandez et al. 2011), chemistry (Sanhueza et al. 2013). See Tan et al. (2014) for a review.

However, there have been fewer studies connecting IRDCs to their larger-scale environments, such as the morphology, 
kinematics and dynamics of their parent clouds. Theories involving production of dense gas in shocks have been supported by detection of large-scale $\mathrm{SiO}$ emission along IRDCs (Jiménez-Serra et al. 2010, Nguyen-Lu'o'ng et al. 2013). However, these studies focus only on a few individual clouds and are still confined to a few-parsec scales in and around the filamentary molecular clouds.

Here we study the ${ }^{13} \mathrm{CO}(1-0)$-emitting gas in and around 10 well-studied IRDCs, utilizing data from the BU-FCRAO Galactic Ring Survey (GRS; Jackson et al.|2006). We consider a range of scales out to 30 pc projected radius, expected to encompass the potential GMC environment of the IRDC. While our study connects to scales typical of other largesample GMC studies (e.g., Heyer et al. 2009, Roman-Duval et al. 2009, 2010), by focusing on just 10 regions we are able to investigate their kinematic properties in much greater detail. Other studies done on such a range of scales, from clump to GMCs, have been performed on nearby GMCs, such as Orion A (e.g., Shimajiri et al. 2011), Taurus (e.g., Goldsmith et al. 2008), and Perseus (Ridge et al. 2006: Foster et al. 2009 Kirk et al. 2010). However, these local GMCs do not seem to give rise to the more extreme range of star-forming clumps that is found in IRDCs.

The main focus of this paper is to "bridge the gap" between IRDC and GMC studies. The questions we aim to address include: Are IRDCs typically found within GMCs? Are IRDCs found within specific locations with respect to GMCs? Are IRDCs and their surrounding GMCs virialized, and does their degree of virialization vary as a function of cloud physical scale?

The IRDC/GMC sample is presented in $\S 2$. Methods for defining cloud boundaries and estimating masses and kinematic properties are described in $\S 3$. Results are presented in $\S 4$, including derived physical properties of the clouds (4.1), the location of IRDCs within their respective GMCs 4.2 , and the kinematic and dynamical analysis of the IRDC and GMCs $(4.3$. We conclude in $\S 5$.

\section{THE IRDC SAMPLE AND MOLECULAR LINE DATA}

We utilize the ${ }^{13} \mathrm{CO}(J=1 \rightarrow 0)$ data of the GRS survey (Jackson et al. 2006), which covers $18^{\circ} \leq l \leq 56^{\circ}, b \pm 1^{\circ}$ and $-5<v_{\mathrm{LSR}}<135 \mathrm{~km} \mathrm{~s}^{-1}$ (for $l \leq 40^{\circ}$ ) and $-5<v_{\mathrm{LSR}}<85 \mathrm{~km} \mathrm{~s}^{-1}$ (for $l \geq 40^{\circ}$ ). The GRS had a spatial resolution of $46^{\prime \prime}$, with $22^{\prime \prime}$ sampling, and a spectral resolution of $0.212 \mathrm{~km} \mathrm{~s}^{-1}$. The typical rms noise is $\sigma\left(\mathrm{T}_{\mathrm{A}}^{*}\right)=0.13 \mathrm{~K}$ (or $\sigma\left(\mathrm{T}_{\mathrm{B}, \nu}\right) \sim 0.26 \mathrm{~K}$ with a main beam efficiency of 0.48$)$.

Our selected IRDCs are the 10 clouds from Butler \& Tan (2009, hereafter BT09) (see also Butler \& Tan 2012, Kainulainen \& Tan 2013). This sample, a subset of that of Rathborne et al. (2006), was chosen while considering the $8 \mu \mathrm{m}$ (IRAC band 4) images from the Spitzer Galactic Legacy Mid-Plane Survey Extraordinaire (GLIMPSE; Benjamin et al. 2003). These IRDCs were selected for being relatively nearby, massive, dark (i.e., relatively high contrast against the surrounding diffuse emission), and surrounded by relatively smooth diffuse emission within the $8 \mu$ m GLIMPSE images. Characteristic sizes and boundaries for each IRDC were taken from Simon et al. (2006a), where ellipses were fitted based on extinction in MSX images. Although these fitted ellipses are not necessarily accurate of IRDC shapes, they provide a convenient measure of the approximate cloud structure. The catalog coordinates and sizes for the 10 IRDCs (including "single component" sub-classifications, labelled "-s", see below) are listed in Table 1.

TABLE 1

The IRDC SAmple

\begin{tabular}{|c|c|c|c|c|c|c|c|c|c|c|c|c|c|}
\hline IRDC & $\left(^{\left.l_{\text {IRDC }}\right)^{\mathrm{a}}}\right.$ & $\begin{array}{c}b_{\text {IRDQ }}{ }^{a} \\
\end{array}$ & $\begin{array}{c}r_{\text {ma }} \\
\left({ }^{\prime}\right) \\
\end{array}$ & $\begin{array}{c}r_{\min }{ }^{a} \\
\left(^{\prime}\right)\end{array}$ & $\begin{array}{c}\mathrm{PA}_{e} \mathrm{a} \\
\left({ }^{\circ}\right)\end{array}$ & $\begin{array}{l}l_{0} \\
\left({ }^{\circ}\right) \\
\end{array}$ & $\begin{array}{l}b_{0} \\
\left(^{\circ}\right) \\
\end{array}$ & $\begin{array}{c}v_{0} \\
\mathrm{~km} \mathrm{~s}^{-1}\end{array}$ & $\begin{array}{l}l_{0, \tau} \\
\left({ }^{\circ}\right) \\
\end{array}$ & $\begin{array}{c}b_{0, \tau} \\
\left({ }^{\circ}\right)\end{array}$ & $\begin{array}{c}v_{0, \tau} \\
\mathrm{km} \mathrm{s}^{-1}\end{array}$ & $\left(\begin{array}{c}b \\
(\mathrm{bpc})\end{array}\right.$ & $\begin{array}{l}T_{\text {ex }}{ }^{\mathrm{c}} \\
(\mathrm{K})\end{array}$ \\
\hline $\mathrm{A}$ & 18.822 & -0.285 & 14.1 & 3.90 & 74.0 & 18.850 & -0.276 & 64.3 & 18.862 & -0.276 & 64.5 & 4.80 & 8.90 \\
\hline B & 19.271 & 0.074 & 8.40 & 1.80 & 88.0 & 19.287 & 0.075 & 26.5 & 19.287 & 0.075 & 26.7 & 2.40 & 7.11 \\
\hline $\mathrm{C}$ & 28.373 & 0.076 & 12.0 & 9.30 & 78.0 & 28.370 & 0.062 & 79.0 & 28.364 & 0.062 & 79.2 & 5.00 & 7.86 \\
\hline D & 28.531 & -0.251 & 20.4 & 5.10 & 60.0 & 28.567 & -0.233 & 78.6 & 28.567 & -0.239 & 80.7 & 5.70 & 7.00 \\
\hline D-s & & & & & & 28.555 & -0.251 & 87.9 & 28.549 & -0.257 & 87.7 & & \\
\hline $\mathrm{E}$ & 28.677 & 0.132 & 14.6 & 4.10 & 103 & 28.672 & 0.130 & 80.7 & 28.684 & 0.130 & 81.3 & 5.10 & 7.00 \\
\hline $\mathrm{F}$ & 34.437 & 0.245 & 5.30 & 2.00 & 79.0 & 34.434 & 0.240 & 57.3 & 34.428 & 0.240 & 57.5 & 3.70 & 6.48 \\
\hline F-s & & & & & & 34.434 & 0.240 & 58.0 & 34.428 & 0.240 & 58.0 & & \\
\hline G & 34.771 & -0.557 & 6.60 & 2.00 & 95.0 & 34.764 & -0.559 & 43.9 & 34.764 & -0.559 & 44.1 & 2.90 & 8.69 \\
\hline G-s & & & & & & 34.770 & -0.559 & 44.4 & 34.764 & -0.559 & 44.4 & & \\
\hline $\mathrm{H}$ & 35.395 & -0.336 & 20.6 & 6.40 & 59.0 & 35.422 & -0.319 & 47.5 & 35.416 & -0.319 & 48.0 & 2.90 & 6.61 \\
\hline H-s & & & & & & 35.391 & -0.337 & 43.9 & 35.391 & -0.337 & 44.4 & & \\
\hline I & 38.952 & -0.475 & 7.50 & 3.00 & 64.0 & 38.942 & -0.473 & 43.5 & 38.942 & -0.473 & 43.2 & 2.70 & 7.24 \\
\hline $\mathrm{J}$ & 53.116 & 0.054 & 1.60 & 1.30 & 50.0 & 53.114 & 0.056 & 21.4 & 53.120 & 0.056 & 21.6 & 1.80 & 7.05 \\
\hline
\end{tabular}

a IRDC coordinates and elliptical sizes adopted from Simon et al. (2006a)

bIRDC kinematic distances adopted from Rathborne et al. (2006)

${ }^{c}$ The ${ }^{13} \mathrm{CO}$ excitation temperatures adopted from Roman-Duval et al. (2010)

Kinematic distances were adopted from Rathborne et al. (2006) and Simon et al. (2006b), where the IRDC central velocity was matched morphologically between the mid-infrared (MIR) extinction estimated from MSX and the ${ }^{13} \mathrm{CO}$ emission from the GRS. These distances were estimated assuming the rotation curve of Clemens (1985). We assume uncertainties of $20 \%$, given the size of streaming motions of $\sim 10-20 \mathrm{~km} \mathrm{~s}^{-1}$ of clouds with trigonometric parallax measurements (e.g., Brunthaler et al. [2009; Reid et al. [2014). 

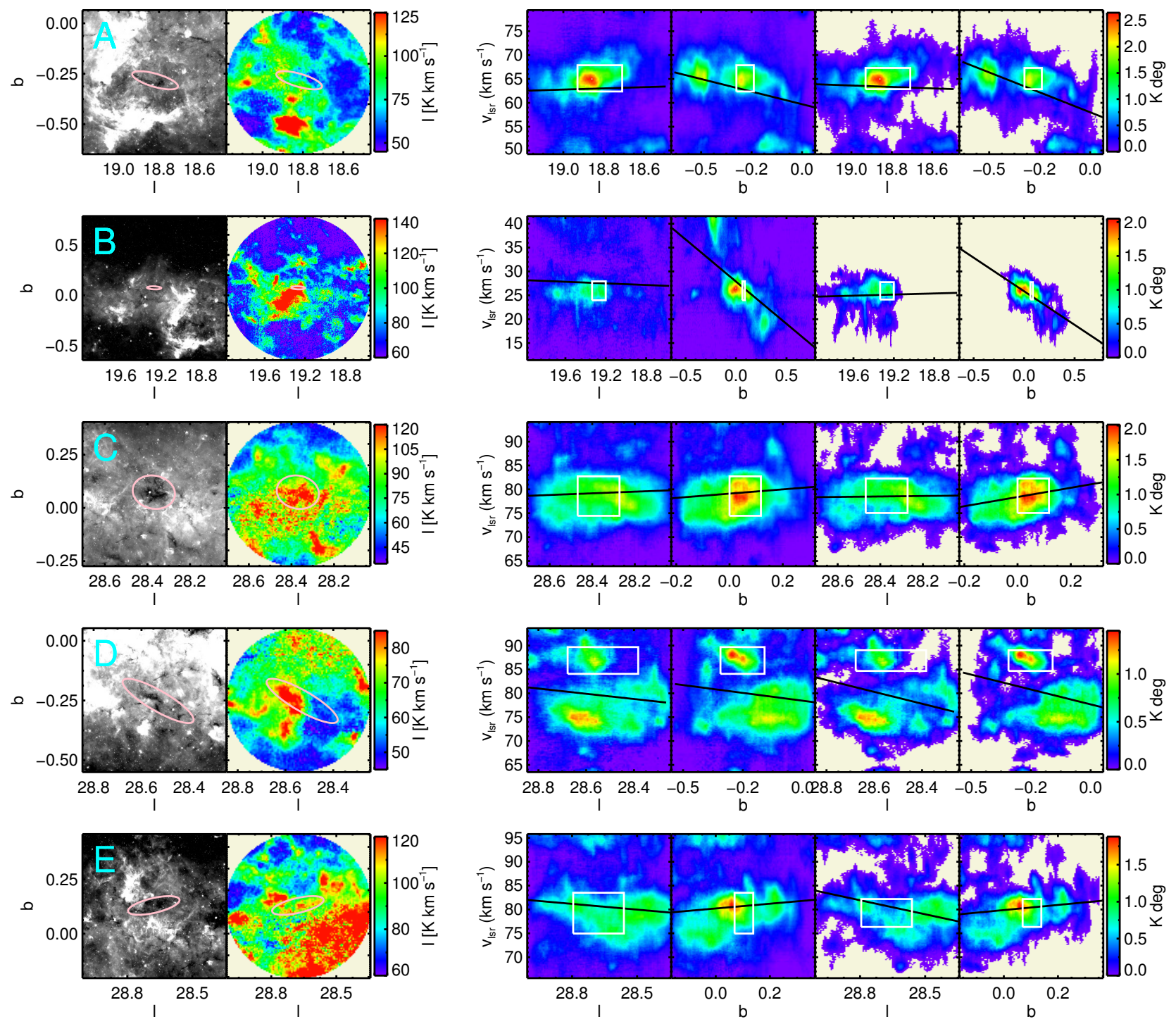

FIG. 1. - The IRDC/GMC sample: each row presents a single cloud (A through E) showing data out to a radius of 30 pc. Left to right: GLIMPSE $8 \mu \mathrm{m}$ image with the IRDC boundary marked with a pink ellipse (see Figs. 3 and A1.A4 for intensity scales); ${ }^{13} \mathrm{CO}(1-0)$ GRS integrated intensity map (over the range $v_{0} \pm 15 \mathrm{~km} \mathrm{~s}^{-1}$ ) in units of $\mathrm{K} \mathrm{km} \mathrm{s}{ }^{-1}$; Full projections of the cloud in position-velocity space: simple-extraction (SE) ${ }^{13} \mathrm{CO}(1-0) \quad v_{\mathrm{lsr}}$ vs $l$ map, $\mathrm{SE}{ }^{13} \mathrm{CO}(1-0) v_{\mathrm{lsr}}$ vs $b$ map; connected extraction $(\mathrm{CE}){ }^{13} \mathrm{CO}(1-0) v_{\mathrm{lsr}}$ vs $l$ map; $\mathrm{CE}$ ${ }^{13} \mathrm{CO}(1-0) v_{\mathrm{lsr}}$ vs $b$ map. The IRDC is marked with a white box of width equal to the extent of the elliptical boundary and height equal to the FWHM of the IRDC co-added spectra. The solid black line shows the mass-weighted linear velocity gradient $\left(d v_{0} / d s\right)$ across each cloud at $R=30 \mathrm{pc}$.

\section{METHODS: DERIVATIONS OF MOLECULAR CLOUD PROPERTIES}

\subsection{Definition of Cloud Boundaries}

We first determined the cloud center-of-mass in position-velocity space. To do this, we co-added the ${ }^{13} \mathrm{CO}$ spectra within an elliptical boundary defined by twice the $r_{\text {maj }}$ and $r_{\text {min }}$ values, using the full GRS velocity range. Then, a velocity interval of $30 \mathrm{~km} \mathrm{~s}^{-1}$ was considered, centered on the peak of the emission profile. The cloud's center-of-mass was evaluated for both optically thin and opacity corrected gas, $\left(l_{0}, b_{0}, v_{0}\right)$ and $\left(l_{0, \tau}, b_{0, \tau}, v_{0, \tau}\right)$, respectively. We then use this as the reference point around which to search for GMC-scale gas, out to a projected radius of 30 pc and over a new velocity range of $v_{0} \pm 15 \mathrm{~km} \mathrm{~s}^{-1}$. This range is wide enough to sample the velocities of GMC gas, even those that are potentially undergoing collisional interactions at velocities set by Galactic shear at the tidal radii of the clouds (Tan 2000, Tasker \& Tan 2009). The GLIMPSE $8 \mu \mathrm{m}$ and GRS ${ }^{13} \mathrm{CO}$ integrated intensity maps centered on these locations are presented in Figures 1 and 2.

We examined ${ }^{13} \mathrm{CO}$ emission within the $v_{0} \pm 15 \mathrm{~km} / \mathrm{s}$ velocity range and within apertures of varying radii from the cloud center-of-mass coordinate. To explore how boundary definitions affect estimated physical properties, we used two different methods to select ${ }^{13} \mathrm{CO}$ line emission associated with the cloud. First, "Simple Extraction (SE)" selected 

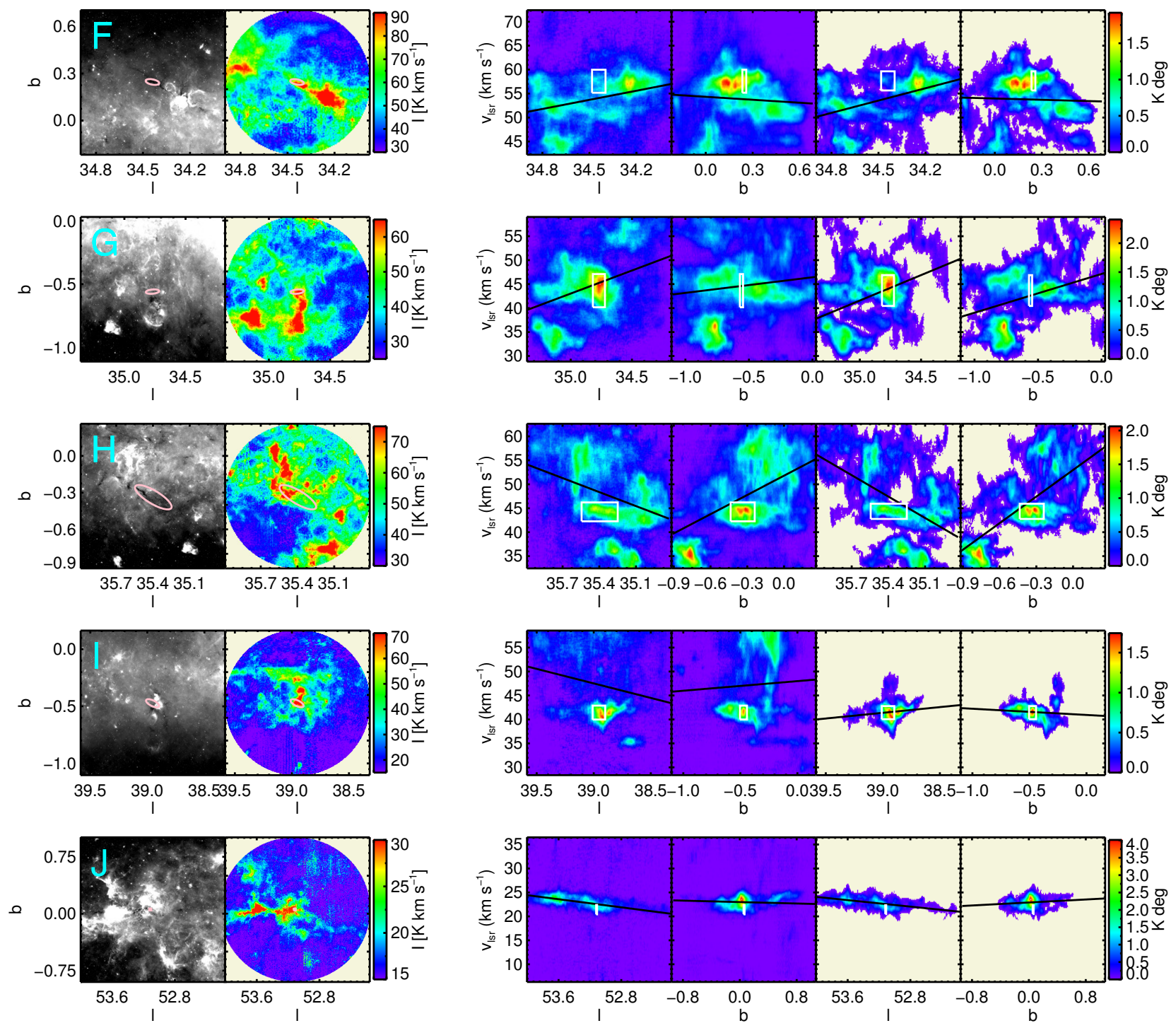

FIG. 2.- The same as Figure 1 but for Clouds F through J.

all the ${ }^{13} \mathrm{CO}$ emission within radii $R=5,10,20$, and $30 \mathrm{pc}$ and $v_{0} \pm 15 \mathrm{~km} / \mathrm{s}$. Second, "Connected Extraction (CE)" defined a cloud as a connected structure in $l-b$ - $v$-space with all cloud voxels required to be above a given threshold intensity: we defined a given voxel as "molecular cloud gas" if its ${ }^{13} \mathrm{CO}(1-0)$ line intensity $T_{B, \nu} \geq 1.35 \mathrm{~K}$ (i.e., the GRS $5 \sigma_{\text {rms }}$ noise level). The CE search was also limited to within a $30 \mathrm{pc}$ radius and $\pm 15 \mathrm{~km} \mathrm{~s}^{-1}$ of the IRDC center-of-mass. Position-velocity maps for each cloud, defined by both SE and CE, are shown in Figures 1 and 2

Clouds defined by CE do not have simple radial sizes. Therefore, we estimated three circular boundaries, centered on the extracted cloud's center-of-mass: (1) mass-weighted radius, $R_{M}$, defined as mean projected radial distance of cloud mass from the center of mass; (2) areal radius, $R_{A}$, defined by the total projected area $A=\pi R_{A}^{2}=N_{p} A_{p}$, where $N_{p}$ is the total number of pixels subtended by the cloud and $A_{p}$ is the area of one image pixel; (3) half-mass radius, $R_{1 / 2}$, defined as the radius from cloud center that contains half of the total mass.

For the IRDCs, we also selected "cloud" material via SE and CE using the spatial coordinates and elliptical boundaries from Simon et al. (2006a), along with the $v_{0} \pm 15 \mathrm{~km} \mathrm{~s}^{-1}$ velocity intervals.

\subsection{Column Densities and Masses from ${ }^{13} \mathrm{CO}$ emission}

We estimated the ${ }^{13} \mathrm{CO}$ column density of each molecular cloud voxel, $d N_{13 \mathrm{CO}}$ from their $J=1 \rightarrow 0$ line emission assuming a partition function with a thermal distribution described by an excitation temperature $T_{\text {ex }}$ via:

$$
\frac{d N_{13 \mathrm{CO}}}{d v}=\frac{8 \pi Q_{\mathrm{rot}}}{A \lambda_{0}^{3}} \frac{g_{l}}{g_{u}} \frac{\tau_{\nu}}{1-\exp \left(-h \nu /\left[k T_{\mathrm{ex}}\right]\right)},
$$


where $Q_{\text {rot }}$ is the partition function, $A=6.294 \times 10^{-8} \mathrm{~s}^{-1}$ is the Einstein coefficient, $\lambda_{0}=0.27204 \mathrm{~cm}, g_{l}=1$ and $g_{u}=3$ are the statistical weights of the lower and upper levels and $\tau_{\nu}$ is the optical depth of the line at frequency $\nu$, i.e., at velocity $v$. Each GRS voxel has a velocity width of $\mathrm{d} v=0.212 \mathrm{~km} \mathrm{~s}^{-1}$. For linear molecules, the partition function is $Q_{\text {rot }}=\sum_{J=0}^{\infty}(2 J+1) \exp \left(-E_{J} / k T_{\mathrm{ex}}\right)$ with $E_{J}=J(J+1) h B$ where $J$ is the rotational quantum number and $B=5.5101 \times 10^{10} \mathrm{~s}^{-1}$ is the rotational constant. For ${ }^{13} \mathrm{CO}(1-0)$ we have $E_{J} / k=5.289 \mathrm{~K}$.

Many studies of the physical properties of GMCs and IRDCs have accounted for line optical depth when estimating their physical properties (e.g., Heyer et al. 2009; Roman-Duval et al. 2010; Hernandez \& Tan 2011; Hernandez et al. 2011, 2012). In Hernandez \& Tan (2011), we showed that optical depth correction factors can increase the ${ }^{13} \mathrm{CO}$ column density by a factor of $\sim 2$ in the densest, sub-parsec scale clumps of IRDCs. However, for the more diffuse GMCs the optical depth correction factors are expected to be smaller. To gauge the importance of this effect, we carry out column density estimates for both the optically thin assumption and accounting for opacity corrections.

The optical depth is evaluated via

$$
T_{B, \nu}=\frac{h \nu}{k}\left[f\left(T_{\mathrm{ex}}\right)-f\left(T_{\mathrm{bg}}\right)\right]\left[1-e^{-\tau_{\nu}}\right],
$$

where $T_{B, \nu}$ is the brightness temperature at frequency $\nu, f(T) \equiv[\exp (h \nu /[k T])-1]^{-1}$, and $T_{\mathrm{bg}}=2.725 \mathrm{~K}$ is the background temperature. $T_{B, \nu}$ is derived from the antenna temperature, $T_{A}$, via $T_{A} \equiv \eta f_{\text {clump }} T_{B, \nu}$, where $\eta$ is the main beam efficiency $\left(\eta=0.48\right.$ for the GRS) and $f_{\text {clump }}$ is the beam dilution factor of the ${ }^{13} \mathrm{CO}$ emitting gas, which we assume to be unity due to the large scale extent of GMCs. Smaller scale structures are undoubtedly present, e.g., as revealed in the BT09 MIR extinction maps, but to gauge the effects of these on the CO emission requires higher resolution molecular line maps of the clouds. For $\tau \ll 1$, Equation (2) can be simplified to express $\tau_{\nu}$ by: $\tau_{\nu}=\left(T_{B, \nu} k /[h \nu]\right)\left[f\left(T_{\mathrm{ex}}\right)-f\left(T_{\mathrm{bg}}\right)\right]^{-1}$. With this simplification, for an observed voxel $T_{B, \nu}$ and an assumed $T_{\mathrm{ex}}$, the optically thin ${ }^{13} \mathrm{CO}$ column density per voxel is given by combining Equations (1) and (2):

$$
\frac{d N_{13 \mathrm{CO}}}{d v}=1.251 \times 10^{14} \frac{Q_{\mathrm{rot}}}{f\left(T_{\mathrm{ex}}\right)-f\left(T_{\mathrm{bg}}\right)} \frac{T_{B, \nu}}{1-\exp \left(-h \nu /\left[k T_{\mathrm{ex}}\right]\right)} \frac{\mathrm{cm}^{-2}}{\mathrm{~km} \mathrm{~s}^{-1}} .
$$

Early studies of IRDCs estimated typical gas kinetic temperatures $T_{\text {gas }} \sim 20 \mathrm{~K}$ (Carey et al. 1998, 2000; Pillai et al. 2006). IRDC F was estimated to have a temperature of $19 \mathrm{~K}$ based on $\mathrm{NH}_{3}(1,1)$ and $(2,2)$ VLA observations (Devine 2009). However, as discussed below, CO excitation temperatures appear to be significantly lower.

In our previous study of IRDC $\mathrm{H}$, we used IRAM 30m observations of $\mathrm{C}^{18} \mathrm{O}(2-1)$ and (1-0) emission from around the IRDC filament to estimate a mean $T_{\mathrm{ex}} \sim 7 \mathrm{~K}$ (Hernandez et al. 2011, hereafter H11). Here, for a uniform analysis of the 10 IRDCs, we now use ${ }^{12} \mathrm{CO} T_{\text {ex }}$ estimates from Roman-Duval et al. (2010, hereafter RD10). In their study of 580 molecular clouds, brightness temperatures from ${ }^{12} \mathrm{CO}(1-0)$ emission line data (Univ. of Massachusetts-Stony Brook (UMSB) Galactic Plane Survey), were used to derive proxy ${ }^{13} \mathrm{CO}$ excitation temperatures, assuming ${ }^{12} \mathrm{CO}$ emission was optically thick and that ${ }^{13} \mathrm{CO}$ and ${ }^{12} \mathrm{CO}$ excitation temperatures are equal. Ultimately, RD10 cited a mean excitation temperature for all their molecular clouds based on all cloud voxels above $4 \sigma_{\mathrm{rms}}$. The RD10 clouds were extracted from the GRS data using a modified version of CLUMPFIND (Williams et al. 1994), which allowed for varying thresholds (i.e., contour increment and minimum brightness, see Rathborne et al. (2009) for details).

Eight of our IRDCs overlapped with at least one of their molecular clouds, and in these cases we adopted $T_{\text {ex }}$ from the RD10 value from the overlapping cloud(s). For the remaining two IRDCs (D and E) we set $T_{\mathrm{ex}}=7 \mathrm{~K}$, similar to the mean values of $7.2 \mathrm{~K}$ of $\mathrm{H} 11$ and $6.32 \mathrm{~K}$ of RD10. Our adopted values of $T_{\mathrm{ex}}$ are listed in Table 1 . For our column density estimates that assume optically thin ${ }^{13} \mathrm{CO}$ emission, these $T_{\mathrm{ex}}$ values are assumed to be constant throughout the cloud. These temperatures are slightly lower (by a few K) than those used in previous studies (e.g., Simon et al. 2001, 2006b who assumed a fixed value of $10 \mathrm{~K}$ ). However, the results from Heyer et al. (2009) indicate that CO gas throughout GMCs is mostly sub-thermally excited. Note that, in this optically thin limit, varying $T_{\text {ex }}$ from $5 \mathrm{~K}$ to $10 \mathrm{~K}$ would change the derived column density of the cloud by only $\sim 20 \%$.

For the opacity-corrected case, the use of a single mean excitation temperature of relatively low value $(\sim 7 \mathrm{~K})$ can lead to non-physical results in certain regions of the cloud. Equation 2 implies $\tau_{\nu}=-\ln \left(1-\left[\left(T_{B, \nu} k /[h \nu]\right)\left[f\left(T_{\mathrm{ex}}\right)-f\left(T_{\mathrm{bg}}\right)\right]^{-1}\right]\right)$. Thus, for a given observed $T_{B, \nu}, \tau_{\nu}$ will become undefined if $T_{\mathrm{ex}} \leq T_{\mathrm{ex}, \text { crit }}$ (i.e., when $\left[\left(T_{B, \nu} k /[h \nu]\right)\left[f\left(T_{\mathrm{ex}}\right)-f\left(T_{\mathrm{bg}}\right)\right]^{-1}\right]>$ 1). For example, a voxel with an observed brightness temperature of $T_{B, \nu}=5 \mathrm{~K}$ will have a numerically undefined opacity at $T_{\text {ex }, \text { crit }}<8.2 \mathrm{~K}$. H11 showed modest temperature variations were present in IRDC $\mathrm{H}$, with a peak temperature of $T_{\mathrm{ex}} \sim 10 \mathrm{~K}$ within the densest clumps and $T_{\mathrm{ex}} \sim 7 \mathrm{~K}$ in the more diffuse gas within the filament envelope (see H11, Fig. 1). Hence, we expect that the voxels containing the largest brightness temperatures have excitation temperatures which are a few $\mathrm{K}$ larger than the constant $T_{\mathrm{ex}}$ values adopted from RD10.

To estimate the opacity-corrected column density, we first apply our adopted $T_{\text {ex }}$ to estimate $\tau_{\nu}$ in each voxel. Then, for each voxel with an undefined $\tau_{\nu}$, we specified a new excitation temperature of $T_{\text {ex,crit }}+1 \mathrm{~K}$, given the voxel's observed $T_{B, \nu}$. This revised excitation temperature allows us to estimate a real solution for $d N_{13 \mathrm{CO}} / d v$ throughout the cloud. After considering a range of possible temperature offsets from $T_{\text {ex,crit }}$, we estimate that the uncertainty in $d N_{13 \mathrm{CO}} / d v$ in an individual voxel by this method is at a level of $\sim 20 \%$.

Ideally, excitation temperatures would be estimated locally from ${ }^{12} \mathrm{CO} J=1-0$ observations of the clouds, assuming that the ${ }^{12} \mathrm{CO}$ line is optically thick. However, there are currently no other ${ }^{12} \mathrm{CO}$ surveys that match both the resolution 
and spatial extent of the GRS. The widely used Columbia-CfA ${ }^{12} \mathrm{CO}$ survey covers the whole Galactic plane, but with a low angular resolution of $8^{\prime}$ (Dame et al. 2001). The RD10 temperatures measurements are based on the UMSB survey, which has a $44^{\prime \prime}$ spatial resolution with $3^{\prime}$ sampling.

For the clouds defined by SE extraction and with $R=30 \mathrm{pc}$, we find that on average $0.7 \%$ of the cloud voxels require higher temperatures than those listed in Table 1. Cloud D has the highest percentage, 1.5\%, and for these voxels $T_{\text {ex,crit }}$ peaks at $16.4 \mathrm{~K}$ with a mean $T_{\text {ex,crit }}=8.3 \mathrm{~K}$. For the clouds defined by CE extraction and $R_{A}$, we find that on average $10 \%$ of voxels require higher excitation temperatures. Here, Cloud J has the highest percentage, $27 \%$, with a peak $T_{\text {ex,crit }}$ of $25.0 \mathrm{~K}$ and a mean $T_{\text {ex,crit }}=9.2 \mathrm{~K}$.

The ${ }^{13} \mathrm{CO}$-based mass per voxel, $d M$ was then calculated by assuming a $n_{12 \mathrm{CO}} / n_{13 \mathrm{CO}}=54$ (Milam et al. 2005) and $n_{12 \mathrm{CO}} / n_{\mathrm{H} 2}=2.0 \times 10^{-4}$ (Lacy et al. 1994). Hence, the assumed abundance of ${ }^{13} \mathrm{CO}$ to $\mathrm{H}_{2}$ is $3.70 \times 10^{-6}$. The mass located at a given voxel is then given by

$$
d M=1.45 \times 10^{-4} \frac{d N_{13 \mathrm{CO}}}{10^{13} \mathrm{~cm}^{-2}} \frac{d v}{0.212 \mathrm{~km} \mathrm{~s}^{-1}}\left(\frac{d}{\mathrm{kpc}}\right)^{2} \frac{\Delta l}{22^{\prime \prime}} \frac{\Delta b}{22^{\prime \prime}} \mathrm{M}_{\odot},
$$

where $d$ is the cloud distance, $\Delta l$ and $\Delta b$ are the angular sizes of the GRS pixels, and assuming a mass per $\mathrm{H}$ nucleus of $\mu_{\mathrm{H}}=2.34 \times 10^{-24} \mathrm{~g}$. The total ${ }^{13} \mathrm{CO}$-derived cloud mass, $M$, is simply the total mass of all cloud voxels within radius $R$ and velocity range $v_{0} \pm 15 \mathrm{~km} \mathrm{~s}^{-1}$. For clouds defined by SE, any pixels with total integrated intensities below $5 \sigma_{\text {rms }}$ were omitted from further analysis.

We estimate an uncertainty of $20 \%$ in $T_{\text {ex }}$ due to its intrinsic variation, which in addition to uncertainties due to intrinsic abundance variations, leads to $\sim 30 \%$ uncertainty in the mass surface density $(\Sigma)$. We thus estimate $\sim 50 \%$ random errors in $M$, after accounting for these uncertainties in $\Sigma$ and the cloud kinematic distance estimates (assumed to be $\sim 20 \%$ ). However, we also anticipate that there could be global systematic uncertainties in $M$ (of the whole cloud sample) of up to a factor of 2 , given the uncertainties in overall absolute ${ }^{13} \mathrm{CO}$ abundance.

\subsection{Cloud Kinematics}

We used co-added ${ }^{13} \mathrm{CO}$ column density velocity distributions (e.g., Fig. 3 . Right) to determine the mean velocity, $v_{0}$ of each extracted cloud. The velocity dispersion was estimated using two standard methods: 1) the rms $1 \mathrm{D}$ velocity dispersion, $\sigma ; 2)$ the width of a fitted Gaussian profile, $\sigma_{G}$. We estimate an mean uncertainty in $\sigma$ of $10 \%$. Additionally, we used these Gaussian fitted profiles to estimate a Gaussian profile mass, $M_{G}$, of each cloud. To visualize how $v_{0}$ and $\sigma$ vary throughout the cloud, we show mass-weighted first and second moment maps of each GMC (e.g., Fig. 3 ).

Using the first moment maps, we derive the velocity gradients in each spatial direction. For example, the longitudinal velocity gradient, $d v_{0} / d l$, was derived by first estimating the mean velocity at each longitudinal position, via a massweighted sum along the perpendicular $(b)$ direction, then finding the best (mass-weighted) linear fit to these velocities. This method was repeated for $d v_{0} / d b$. The magnitude of the total linear velocity gradient across the cloud, $d v_{0} / d s$, and its position angle direction, $\theta_{v}$, were then calculated. Note, we choose to use mass-weighted velocity gradients to prevent the results being unduly affected by tenuous wisps of cloud material. Also, in the context of interpreting velocity gradients as due to rotation and thus measuring rotational energies of the cloud (below), this mass-weighted gradient is the appropriate one to use.

Many studies of the dynamics of molecular clouds have interpreted total GMC linear velocity gradients as due to solid body rotation (e.g., Phillips 1999, Rosolowsky et al. 2003: Imara \& Blitz 2011). However, the possibility remains that the identified single "cloud" actually consists of spatially independent structures. For a cloud undergoing solid body rotation, the line of sight velocity gradient in the plane of the sky, $d v_{0} / d s$, is equal to the projected angular velocity, i.e., $\Omega_{0}=d v_{0} / d s$. The true angular velocity is $\Omega=\Omega_{0} / \sin i$, where $i$ is the angle between the rotation axis and the line of sight.

The position angle of the projected rotation axis of the cloud contains information that may constrain theories of GMC formation and evolution. For example, if a GMC forms rapidly from atomic gas in the Galactic plane, then the GMC rotation is expected to be prograde with respect to Galactic rotation (e.g., Tasker \& Tan 2009). If strong gravitational encounters and collisions are frequent between gravitationally bound GMCs (Tan 2000), then a more random set of orientations of the positional angles of projected rotation axes are expected, including both pro- and retrograde rotating clouds. For clouds observed in the Galactic plane, $-90^{\circ}<\theta_{v}<+90^{\circ}$ represents retrograde rotation and $-90^{\circ}<\theta_{v}<-180^{\circ}$ and $90^{\circ}<\theta_{v}<180^{\circ}$ represent prograde rotation.

We then also estimated the projected moment of inertia, $I_{0}=\sum d M_{i} s_{i}^{2}$, of each cloud using the sky projected rotation axis, defined by $\theta_{v}$ and the cloud center-of-mass coordinate, where $s_{i}$ is the shortest distance to the rotation axis. This allows estimation of the projected rotational energy of the cloud, $E_{\mathrm{rot}, 0}=(1 / 2) I_{0} \Omega_{0}^{2}$.

\section{RESULTS}

\subsection{Molecular Cloud Physical Properties}

Table 2 presents the derived physical properties for all 10 clouds. The global properties of each cloud were evaluated for data extracted out to seven different radii, shown on different lines in the table: $5,10,20$, and 30 pc using SE; and out to mass weighted $\left(R_{M}\right)$, areal $\left(R_{A}\right)$, and half-mass $\left(R_{1 / 2}\right)$ radii using CE. Each cloud definition case is noted in column 1. The center-of-mass coordinates of each cloud are listed in columns 2 and 3 , evaluated for the optically thin and opacity-corrected cases. We note that the $\mathrm{CE}$ cloud definitions with $R_{M}, R_{A}$ and $R_{1 / 2}$ share the same 


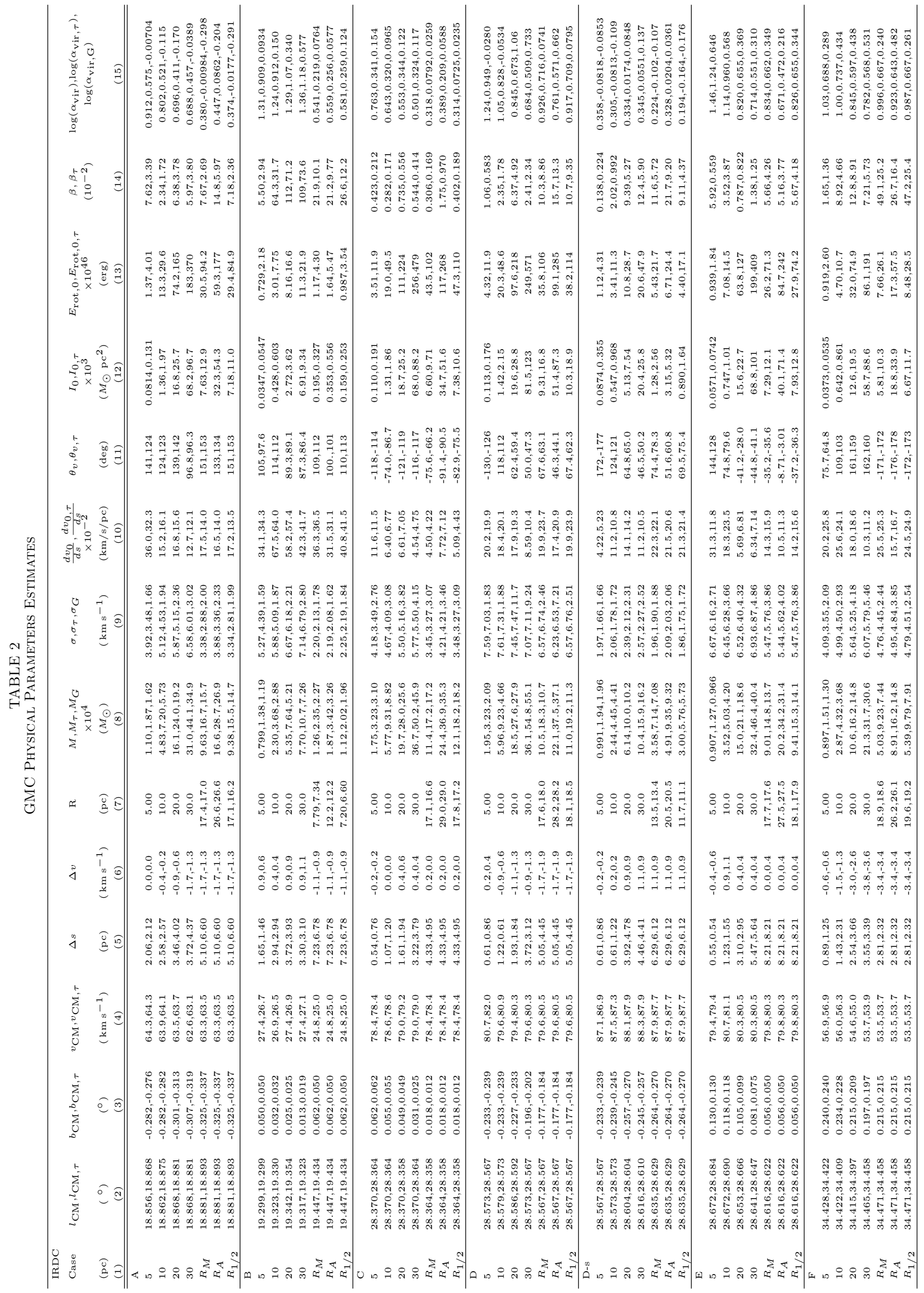




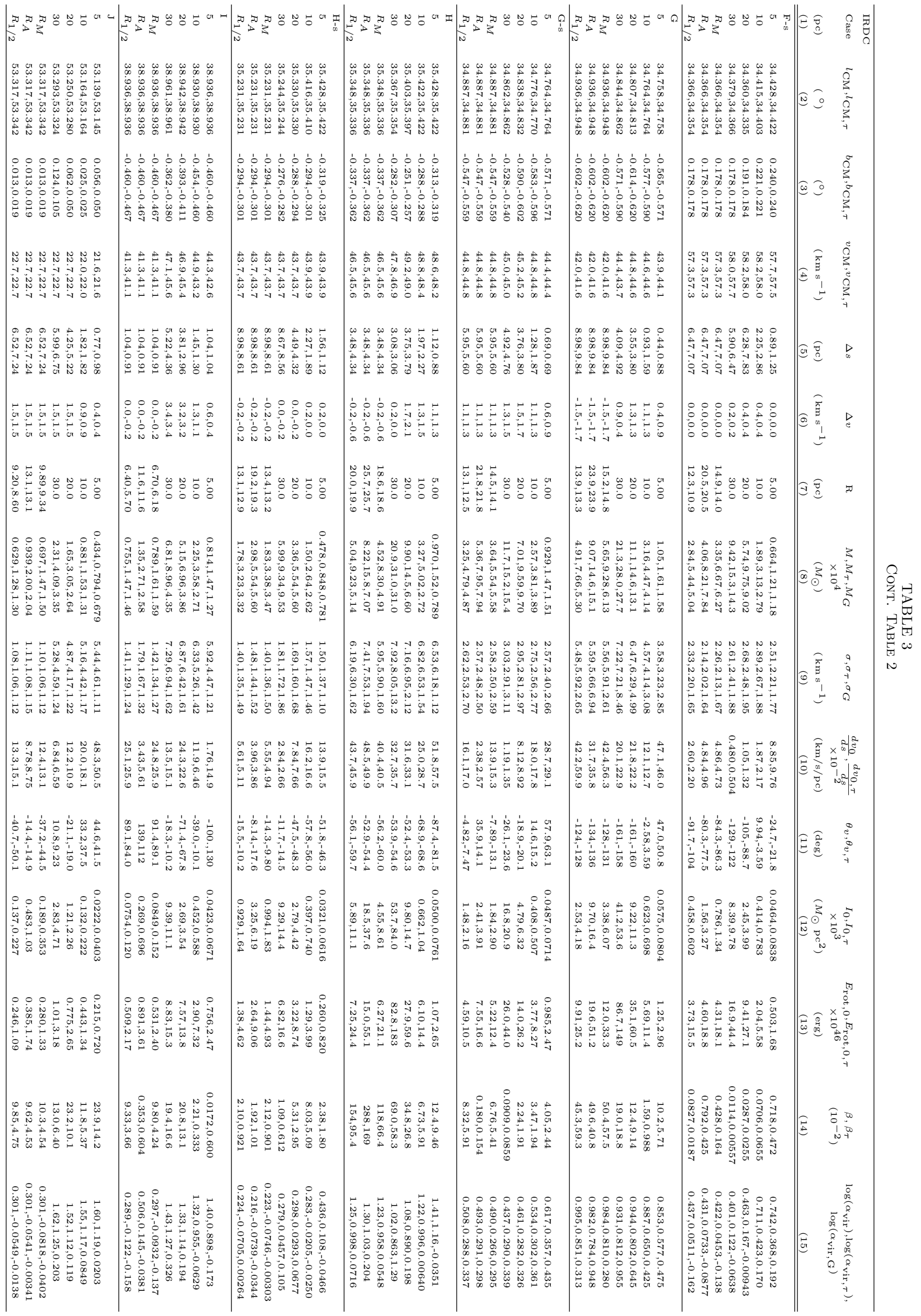




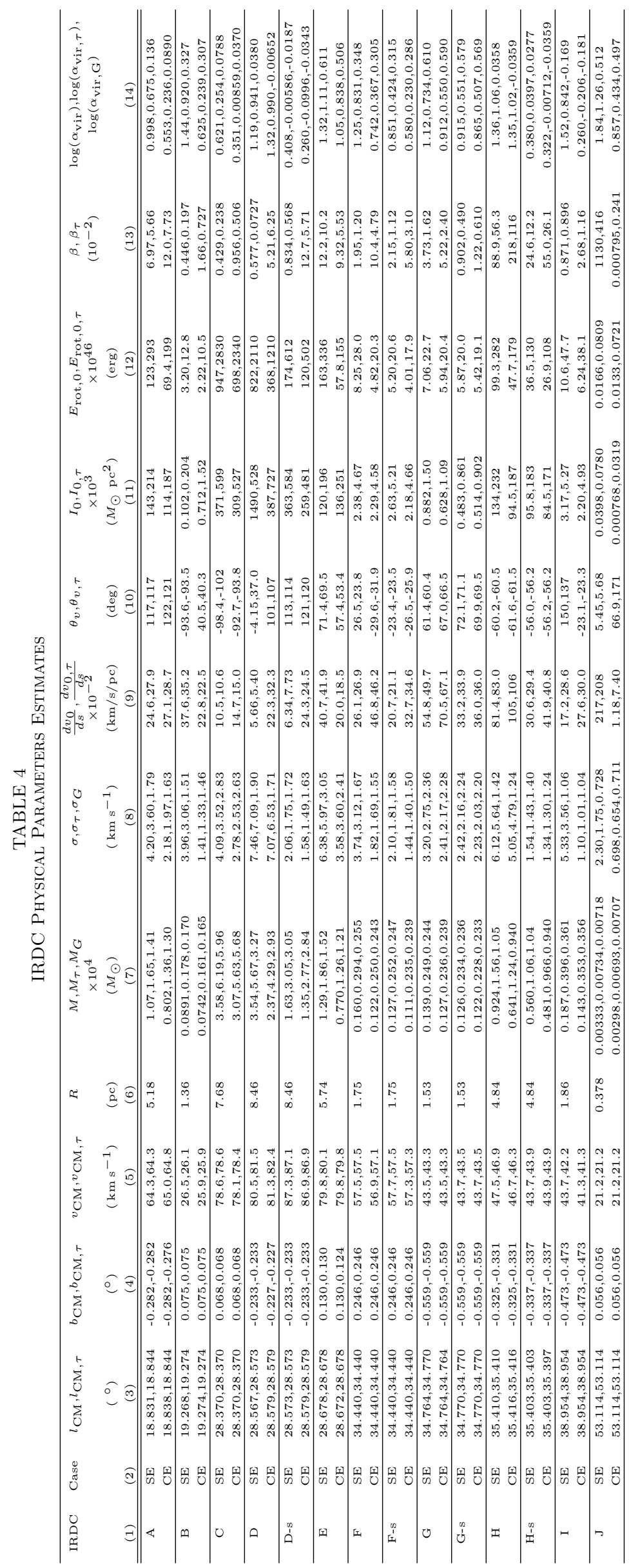


center. Mean velocities are reported in column 4. Columns 5 and 6 list the separations between the GMC centers and the IRDC centers in plane of sky distance and velocity offset, respectively, again for both optically thin and opacity-corrected methods. Column 7 lists the cloud radii (with $R_{M}, R_{A}$ and $R_{1 / 2}$ cases having two values for the optical thin and opacity-corrected cases). Column 8 lists the three cloud mass estimates: optically thin mass $(M)$, opacity-corrected mass $\left(M_{\tau}\right)$, and the mass estimated from a gaussian fit of the co-added opacity corrected column density spectra $\left(M_{G}\right)$. Column 9 lists the three velocity dispersions measured: the standard deviations of the cloud's optically thin and opacity corrected column density spectra $\left(\sigma, \sigma_{\tau}\right)$ and the dispersion estimated from the gaussian fitted opacity corrected column density spectra $\left(\sigma_{G}\right)$. Column 10 presents the two velocity gradient magnitudes, one estimated from the optically thin column density weighted first-order moment map $\left(d v_{0} / d s\right)$ and one estimated from the opacity-corrected column density weighted first-order moment map $\left(d v_{0, \tau} / d s\right)$. Column 11 presents the position angles of the projected cloud rotation axes estimated from each cloud's angular momentum for both optically thin and opacity corrected masses $\left(\theta_{v}\right.$ and $\left.\theta_{v, \tau}\right)$. The projected moments of inertia estimated using the optically thin mass $\left(I_{0}\right)$ and opacity corrected mass $\left(I_{0, \tau}\right)$ are presented in column 12 . Column 13 shows projected rotational energies, $E_{\text {rot, }, 0}$ and $E_{\mathrm{rot}, 0, \tau}$, and column 14 shows the ratios of these to the gravitational energy, i.e., $\beta$ and $\beta_{\tau}$. Finally, column 15 shows $\log _{10}$ of the virial parameters of the clouds using the three measures of mass, i.e., $\alpha_{\mathrm{vir}}, \alpha_{\mathrm{vir}, \tau}, \alpha_{\mathrm{vir}, \mathrm{G}}$.

A visual overview of each GMC-scale region is given in Figure 3 and Figures A1 A9. The left side of the figures display a series of images of the molecular gas out to a radius of $30 \mathrm{pc}$. The first row displays the GLIMPSE $8 \mu \mathrm{m}$ image and the ${ }^{13} \mathrm{CO}$ mass surface density map, $\Sigma_{13 \mathrm{CO}}$, generated using the velocity interval given solely by the IRDC. The IRDC elliptical boundary is also shown (Simon et al. 2006a). The subsequent rows display the $\Sigma_{13 \mathrm{CO}}$ maps assuming our defined velocity interval of $v_{0} \pm 15 \mathrm{~km} \mathrm{~s}^{-1}$, the column-density weighted mean velocity (first moment), the linear momentum map derived from each pixel's column density and velocity with respect to the cloud center of mass, and the velocity dispersion map (second moment) for the SE cloud (Left column) and CE cloud (Right column). The right side of the figures display the optically thin and opacity-corrected co-added column density velocity spectra at various extraction radii for the SE and CE clouds and the SE IRDC.

Four of the clouds (D, F, G, and $\mathrm{H}$ ) were found to contain multiple velocity components, based on the co-added ${ }^{13} \mathrm{CO}$ column density spectra of their SE 60 pc diameter GMCs (e.g., Cloud D; Fig. A3). We used a series of integrated intensity maps to isolate the velocity range of the molecular gas associated with the IRDC (Fig. 45). In general, these velocity ranges span $\sim 10 \mathrm{~km} \mathrm{~s}^{-1}$ (Cloud D: $84.1-93.7 \mathrm{~km} \mathrm{~s}^{-1}$; Cloud F: $54.3-65.5 \mathrm{~km} \mathrm{~s}^{-1}$; Cloud G: $39.7-51.8 \mathrm{~km} \mathrm{~s}^{-1}$; Cloud H: $\left.39.7-49.9 \mathrm{~km} \mathrm{~s}^{-1}\right)$. To investigate how the cloud's derived physical properties were affected by isolating only these velocity ranges, we include these four cases as separate entries in Table 2 (noted with "-s"). For all subsequent analyses, the "-s" values for these four clouds are used in place of those derived using the full velocity range of $v_{0} \pm 15 \mathrm{~km} \mathrm{~s}^{-1}$.

\subsection{IRDC and GMC Morphologies}

\subsubsection{Description of Individual IRDCs/GMCs}

Cloud A: We estimate a cloud areal radius of $R_{A} \simeq 27 \mathrm{pc}$ and a mass of $M_{G, A} \simeq 2.7 \times 10^{5} \mathrm{M}_{\odot}$. The GRS ${ }^{13} \mathrm{CO}$ GMC study by RD10 identified five molecular clouds within the extent of Cloud A, with a total ${ }^{13} \mathrm{CO}$ mass of $2.1 \times 10^{5} \mathrm{M}_{\odot}$ (note, we scaled all ${ }^{13} \mathrm{CO}$ masses of RD10 by a factor 0.49 to convert to our assumed ${ }^{13} \mathrm{CO} / \mathrm{H}_{2}$ abundance ratio; see 3.2). IRDC A is located about 20 pc north of its parent GMC's highest $\Sigma$ clump, which appears bright at $8 \mu \mathrm{m}$ and is thus likely to be star-forming. The WISE H II region catalog (Anderson et al. 2014) lists the W39 H II region to be within $\sim 3^{\prime}$ of this clump's center, and with a similar LSR velocity $\left(v_{\mathrm{LSR}}=65.5 \mathrm{~km} \mathrm{~s}^{-1}\right)$. Another H II region lies in a smaller dense clump at $l=19.07^{\circ}, b=-0.28^{\circ}$ with velocity and distance consistent with those of Cloud A. The isolated, simple ${ }^{13} \mathrm{CO}$ emission profiles, along with the spatial connection between the IRDC and the H II regions, demonstrated through CE, indicates that GMC A contains clumps at a variety of stages of star formation activity.

Cloud B: We estimate a radius of $R_{A} \simeq 12 \mathrm{pc}$ and a mass of $M_{G, A} \simeq 3.3 \times 10^{4} M_{\odot}$. RD10 estimated a ${ }^{13} \mathrm{CO}$ mass of $2.4 \times 10^{4} \mathrm{M}_{\odot}$ and a smaller projected areal radius of $6.3 \mathrm{pc}$. The IRDC is located within the densest region of the GMC, which extends $\sim 10 \mathrm{pc}$ to the south-east of the IRDC and accounts for most of the cloud's mass. Spatially, Cloud B is well defined and completely isolated at an extraction radius of $20 \mathrm{pc}$ in CE. Although the cloud is isolated in velocity space, the column density profile indicates a low level of ${ }^{13} \mathrm{CO}$ emission at neighboring velocities, both lower and higher. Although the GLIMPSE $8 \mu \mathrm{m}$ emission map indicates a large bright emission structure to the west of the cloud, this feature is not at velocities consistent with Cloud B $\left(v_{\mathrm{LSR}} \sim 50 \mathrm{~km} \mathrm{~s}^{-1}\right)$.

Cloud $C$ : We estimate a GMC radius of $R_{A} \simeq 29 \mathrm{pc}$ and a mass of $M_{G, A} \simeq 3.5 \times 10^{5} M_{\odot} . \mathrm{RD} 10$ derived a radius of $20.9 \mathrm{pc}$ and a mass of $\sim 3 \times 10^{5} \mathrm{M}_{\odot}$. IRDC $\mathrm{C}$ is located within the dense central region of its parent GMC. The integrated intensity maps indicate dense and clumpy structure throughout the cloud (Fig. A2). The velocity distribution shows a broad centrally-peaked profile, but with potential substructure. Additionally, there is a low level of ${ }^{13} \mathrm{CO}$ emission at higher velocities. Butler et al. (2014) studied the IRDC C using near- and mid-infrared extinction mapping to probe the high-dynamic range of its mass surface density (down to $A_{V} \sim 1 \mathrm{mag}$ ), finding an IRDC mass of $\sim 7 \times 10^{4} M_{\odot}$. This is one of the most massive, high- $\Sigma$ IRDCs known, with the potential to form a massive star cluster. Using the same IRDC elliptical boundary from(Simon et al. (2006a), we estimate a ${ }^{13} \mathrm{CO}$ mass of $5.7 \times 10^{4} M_{\odot}$. Our slightly lower mass estimate may be the result of abundance uncertainties and/or CO depletion.

Cloud D: For the GMC at $v_{\mathrm{LSR}}=84.1-93.7 \mathrm{~km} \mathrm{~s}^{-1}$, we estimate a radius of $R_{A} \simeq 21 \mathrm{pc}$ and a mass of $M_{G, A} \simeq$ $9.3 \times 10^{4} M_{\odot}$. RD10 identified two clouds within the Cloud D-s region with a total ${ }^{13} \mathrm{CO}$ mass of $\sim 5.2 \times 10^{4} \mathrm{M}_{\odot}$. 


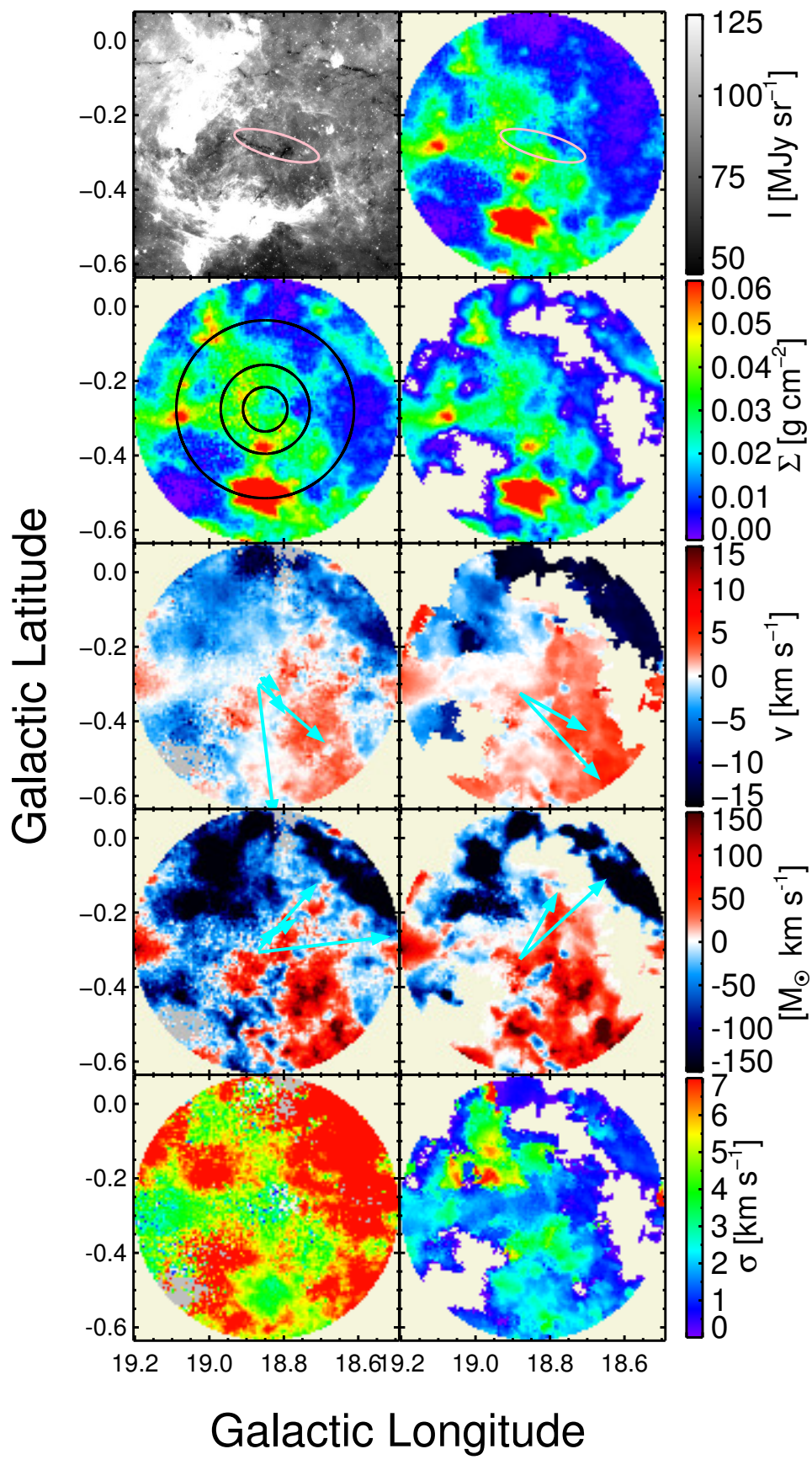

\section{Cloud A}

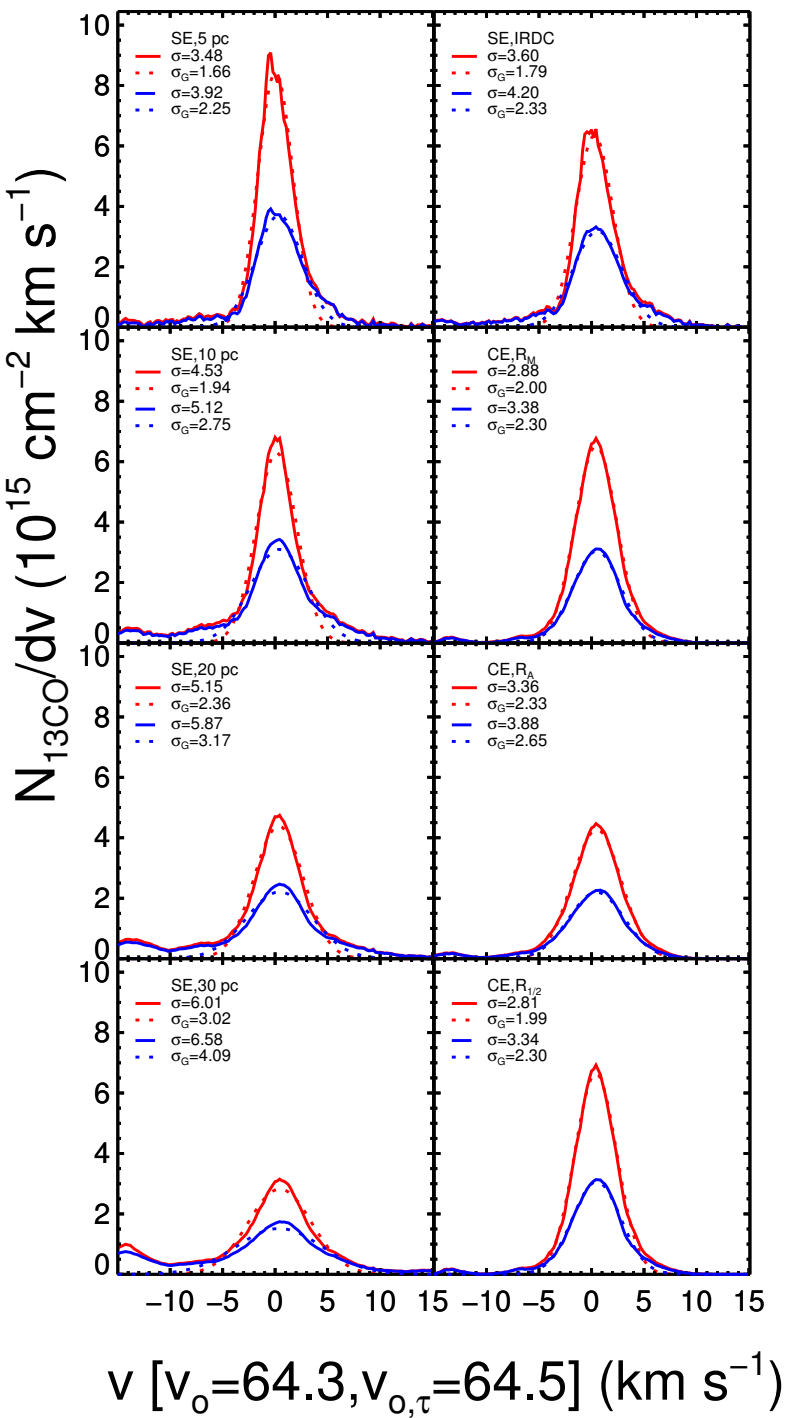

Fig. 3.- Cloud A: Left panel maps: Top row: Left: GLIMPSE $8 \mu \mathrm{m}$ image. Right: ${ }^{13} \mathrm{CO}$ mass surface density $(\Sigma)$ map over the velocity range of the IRDC emission profile $v_{\mathrm{lsr}}=54.6-77.1 \mathrm{~km} \mathrm{~s}^{-1}$ (ellipse denotes IRDC boundary). 2nd row: Left: SE ${ }^{13} \mathrm{CO}-\mathrm{derived}$ $\Sigma$ map (circles denote 5, 10, 20 pc boundaries). Right: $\mathrm{CE}{ }^{13} \mathrm{CO}$-derived $\Sigma$ map. 3rd row: Left: SE ${ }^{13} \mathrm{CO}$ mean velocity (first moment) map. Right: $\mathrm{CE}{ }^{13} \mathrm{CO}$ mean velocity (first moment) map relative to $v_{0}$ defined from the IRDC. The arrows show the direction of total (mass-weighted) line-of-sight velocity gradients, $d v_{0} / d s$. An arrow is shown for each extraction radius (defining the length of the arrow) and is centered on either the extracted cloud's center of mass (for SE cases) or the $R=30$ pc cloud's center of mass (for CE cases). 4th row: Left: $\mathrm{SE}{ }^{13} \mathrm{CO}$-derived (optically thin) linear momentum map. Right: $\mathrm{CE}{ }^{13} \mathrm{CO}$-derived (optically thin) linear momentum map. The arrows show the position angle, $\theta_{v}$, of the cloud's rotation axis, i.e., orthogonal to the direction of the velocity gradient, for each extracted cloud (with arrow length showing cloud radius) 5th row: Left: $\mathrm{SE}{ }^{13} \mathrm{CO}$ velocity dispersion (second moment; optically thin) map. Right: $\mathrm{CE}{ }^{13} \mathrm{CO}$ velocity dispersion (second moment; optically thin) map. All maps in Rows $2-5$ cover the velocity interval of $v_{0} \pm 15 \mathrm{~km} \mathrm{~s}{ }^{-1}$,

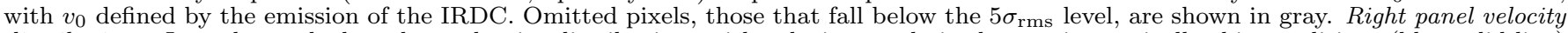
distributions: In each panel, the column density distributions with velocity are derived assuming optically thin conditions (blue solid line) or with opacity correction (red solid line) from the ${ }^{13} \mathrm{CO}(1-0)$ co-added spectra. Gaussian fits are shown by the dotted blue and red lines. In all panels, $\sigma$ and $\sigma_{G}$ are noted in the upper left corner of the plot. Left column: Top to bottom: SE out to $R=5,10,20,30$ pc. Right column: Top to bottom: $\mathrm{SE}$ of IRDC ellipse, CE out to $R_{M}, R_{A}, R_{1 / 2}$. 

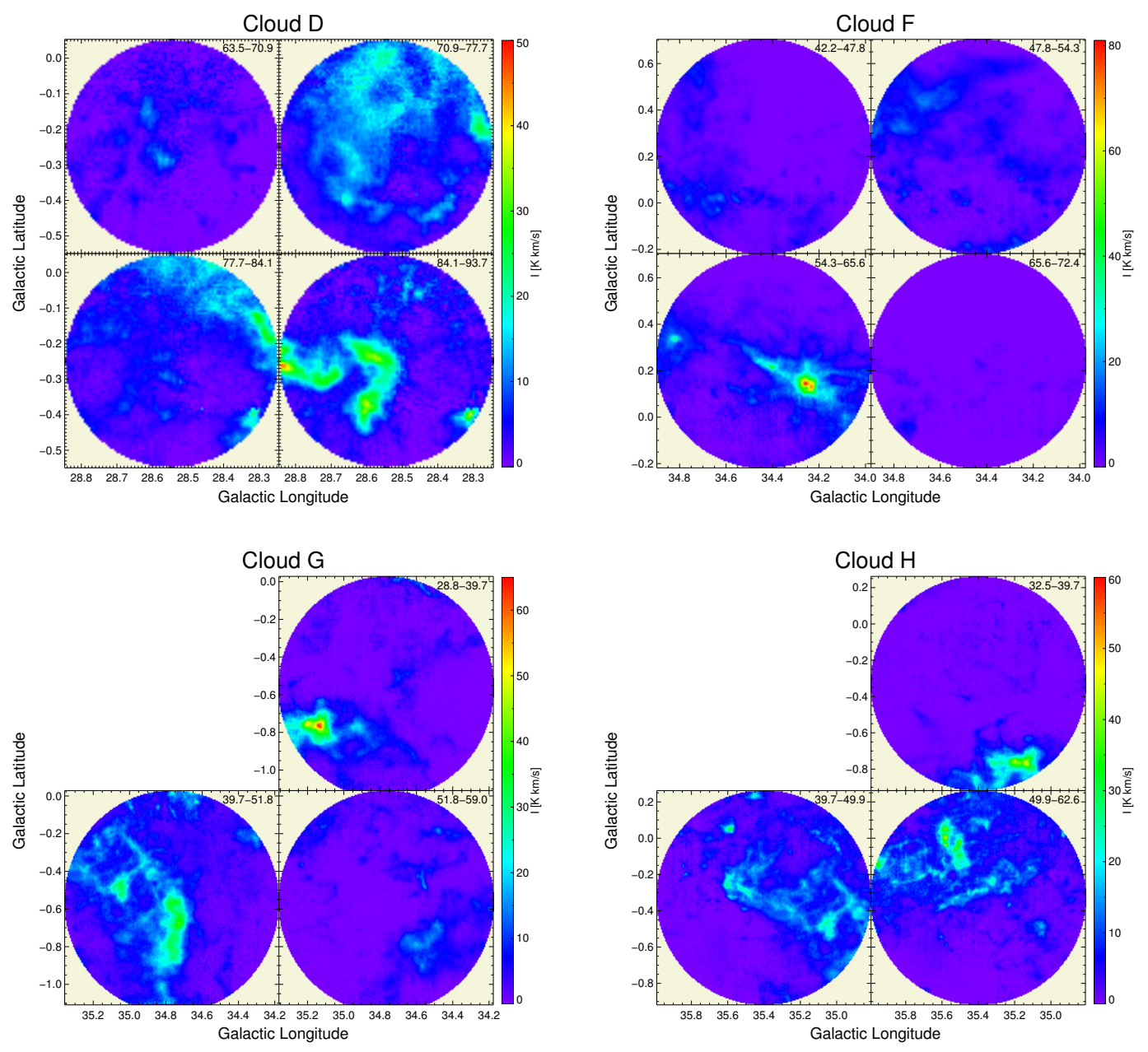

FIG. 4.- ${ }^{13} \mathrm{CO}$ channel maps of the multi-component clouds. top left: Cloud D; top right: Cloud F; bottom left Cloud G; bottom right: Cloud $\mathrm{H}$. The velocity interval of each panel is shown in the upper-right corner of each map.

The IRDC is located in the dense central region of the GMC. However, the ${ }^{13} \mathrm{CO}$ spectra show four cloud components within $v_{0} \pm 15 \mathrm{~km} \mathrm{~s}^{-1}$. Although the ${ }^{13} \mathrm{CO}$ emission corresponding to the IRDC is evident within a radius of $5 \mathrm{pc}$, a lower-velocity gas component begins to dominate on scales out to about 20 pc. The position-velocity map (Fig. 1) shows there is a significant amount of ${ }^{13} \mathrm{CO}$ emission at lower velocities, $v_{\mathrm{LSR}}=63.5-84.1 \mathrm{~km} \mathrm{~s}{ }^{-1}$, that extends largely over the northern region of the cloud. In the same velocity interval of the IRDC, $v_{\mathrm{LSR}}=84.1-93.7 \mathrm{~km} \mathrm{~s}{ }^{-1}$, a substantial molecular gas clump contains the largest integrated peak intensity $\left(\sim 50 \mathrm{~K}^{\mathrm{km} \mathrm{s}} \mathrm{s}^{-1}\right)$ at the western edge of the $30 \mathrm{pc}$ extraction radius. Figure 4 suggests that this emission is only tenuously connected to the ${ }^{13} \mathrm{CO}$ emission associated with the main GMC.

Cloud E: We estimate a GMC radius of $R_{A} \simeq 28 \mathrm{pc}$ and a mass of $M_{G, A} \simeq 3.1 \times 10^{5} M_{\odot}$. No clouds within the Cloud E spatial and velocity ranges were listed in the RD10 catalog. The integrated emission map indicates that the GMC is highly substructured. The IRDC is contained within a small dense clump central to its parent GMC, but more massive clumps are seen on scales beyond 5 pc. CE finds an extended region of clumpy ${ }^{13} \mathrm{CO}$ gas in the cloud's south-west region, beginning at $10 \mathrm{pc}$ and extending beyond the $30 \mathrm{pc}$ boundary.

Cloud F: For the cloud in the velocity range $v_{\mathrm{LSR}}=54.3-65.6 \mathrm{~km} \mathrm{~s}^{-1}$, we estimate a radius of $R_{A} \simeq 21 \mathrm{pc}$ and a mass of $M_{G, A} \simeq 7.8 \times 10^{4} M_{\odot}$, assuming a distance of $3.7 \mathrm{kpc}$ (Rathborne et al. 2006). Note, Kurayama et al. (2011) estimated a parallax distance $1.56 \pm 0.12 \mathrm{kpc}$, however this result has been called into question by Foster et al. $(\overline{2012})$. RD10 found two GMCs associated with Cloud F: one cloud matches the location and velocity interval of Cloud F's western massive clump, having a mass of $\simeq 6.9 \times 10^{4} M_{\odot}$ and radius of 12.3 pc. The other molecular cloud is associated with the smaller eastern clump, for which they estimate a mass of $\sim 2.2 \times 10^{4} \mathrm{M}_{\odot}$. Our analysis recovers this larger total mass, $\sim 10^{5} M_{\odot}$, if we include all the ${ }^{13} \mathrm{CO}$ emission within $v_{0} \pm 15 \mathrm{~km} \mathrm{~s}^{-1}$. The larger molecular cloud complex surrounding Cloud F was recently cataloged within the Giant Molecular Filament (GMF) study of Ragan et al. (2014). They found that their filament GMF38.1-32.4 is actually the projection of two overlapping filamentary structures. Cloud F is within the further filament (3.0-3.7 kpc), which has a total length of 232 pc. The GMF contains multiple infrared dark regions (identified in both GLIMPSE $8 \mu \mathrm{m}$ and HiGAL $250 \mu \mathrm{m}$ images) and several dense gas 
clumps (identified by the Wienen et al. (2012) $\mathrm{NH}_{3}$ and Bolocam Galactic Plane surveys), demonstrating that overall it is in an early phase of star formation. However, in Cloud $\mathrm{F}$ alone, there are two $\mathrm{H}$ II regions within the two densest parts of the cloud with consistent LSR velocities and distances, at $l=34.256, b=0.136$ and $l=34.821, b=0.351$ (Anderson et al. 2014). While IRDC F, which is also highly filamentary, contains some of the highest column densities in the GMC, the highest $\Sigma$ clump is actually another feature extending west of the IRDC between 5 and 25 pc (Fig. A5). The velocity distribution of the GMC for material out to $30 \mathrm{pc}$ indicates that there are four cloud components within our defined velocity interval $v_{0} \pm 15 \mathrm{~km} \mathrm{~s}^{-1}$. Figure 2 shows the PV maps of the cloud out to $30 \mathrm{pc}$ and indicates that the majority of the ${ }^{13} \mathrm{CO}$ emission resides within the velocity interval of the IRDC $\left(v_{\mathrm{LSR}}=54.3-65.6 \mathrm{~km} \mathrm{~s}^{-1}\right)$ and is connected to the weaker molecular emission at surrounding velocities. Additionally, the massive clump and IRDC are kinematically associated with another dense clump on the eastern edge of the cloud at $\sim 25$ pc. The cloud defined by $\mathrm{CE}$ out to a radius of $30 \mathrm{pc}$ indicates that the regions above and below the IRDCs parent cloud are filled with clumpy molecular material related to the other three emission profiles.

Cloud $G$ : For the material in the velocity range of $v_{\mathrm{lsr}}=39.7-51.8 \mathrm{~km} \mathrm{~s}^{-1}$ we estimate a cloud radius of $R_{A} \simeq 22 \mathrm{pc}$ and a mass of $M_{G, A} \simeq 8.0 \times 10^{4} M_{\odot}$. RD10 identified two clouds within the Cloud G boundary with a total ${ }^{13} \mathrm{CO}$ mass of $\sim 5.7 \times 10^{4} M_{\odot}$. At small cloud radii, $<10 \mathrm{pc}$, Cloud G's velocity distribution is reasonably well described by a single peak. However, at larger radii we clearly see three separate components within $v_{0} \pm 15 \mathrm{~km} \mathrm{~s}^{-1}$. The IRDC, and its parent GMC, are confined to the central velocity interval of $v=39.7-51.8 \mathrm{~km} \mathrm{~s}^{-1}$. In the GMC defined by SE, the IRDC lies at the northern edge of the cloud's densest region, which extends south $\sim 18 \mathrm{pc}$. For the cloud defined by $\mathrm{CE}$, this same cloud region breaks up into three smaller clumps. The lower velocity component of the cloud contains a dense molecular clump at $l \simeq 35.2, b \simeq-0.7$, a region which also contains a high level of mid-infrared emission at $8 \mu \mathrm{m}$ (Fig. A6). The WISE H II region database lists a candidate H II region near the center of the low-velocity clump with an estimated parallax distance of $2.2 \mathrm{kpc}$, versus the Cloud G distance of $2.9 \mathrm{kpc}$.

Cloud H: For gas in the velocity range of $v_{\mathrm{lsr}}=39.7-47.1 \mathrm{~km} \mathrm{~s}^{-1}$, we estimate a cloud radius of $R_{A} \simeq 19 \mathrm{pc}$ and a mass of $M_{G, A} \simeq 5.6 \times 10^{4} M_{\odot}$. RD10 identified three molecular clouds at the location of Cloud $\mathrm{H}$, with a total mass of $\simeq 2.8 \times 10^{4} M_{\odot}$. The PV diagrams indicate that there are three separate cloud components within $v_{0} \pm 15 \mathrm{~km} \mathrm{~s}^{-1}$ (Fig. 2). The IRDC and its parent GMC are confined to the velocity interval of $v=39.7-47.1 \mathrm{~km} \mathrm{~s}^{-1}$, in which the IRDC is the main structure of a larger diffuse cloud extending to the west. There are additional dense gas clumps at the neighboring velocities: a large coherent clump about $25 \mathrm{pc}$ to the south at $v=32.5-39.7 \mathrm{~km} \mathrm{~s}^{-1}$ (part of Cloud G), and a high concentration of dense, clumpy gas along the entire northern region at velocities of $v=47.1-62.6 \mathrm{~km} \mathrm{~s}^{-1}$. These higher velocity clumps overlap with a region of bright $8 \mu \mathrm{m}$ dust emission (Fig. A77). The WISE H II database lists five H II regions overlapping these dense clumps with similar LSR velocities (Anderson et al. 2014).

Cloud I: We find that GMC I has a radius of $R_{A} \simeq 12 \mathrm{pc}$ and a mass of $M_{G, A} \simeq 2.6 \times 10^{4} M_{\odot}$. However, RD10 identified two clouds that match the location of Cloud I, and estimated the much larger mass of $\sim 2.4 \times 10^{5} \mathrm{M}_{\odot}$. This difference is due to the surrounding diffuse ${ }^{13} \mathrm{CO}$ emission, which is included by their cloud finding method that uses the CLUMPFIND algorithm (Rathborne et al. 2009). IRDC I lies within the densest region of the GMC, which extends roughly $7 \mathrm{pc}$ north of the IRDC. The cloud's SE column density spectra indicates a symmetric and well isolated emission profile out to radii of $\sim 10 \mathrm{pc}$. However, at larger cloud radii, diffuse ${ }^{13} \mathrm{CO}$ gas at higher velocities is seen. However, the spectra of the cloud defined by CE indicates that this high-velocity emission is at intensities less than the $5 \sigma$ threshold, suggesting that the GMCs is surrounded by a reservoir of low-density molecular gas. For the cloud defined by $\mathrm{CE}$, the central dense region is broken down into three dense clumps and the GLIMPSE $8 \mu \mathrm{m}$ image indicates that there are multiple bright dust regions along these dense ${ }^{13} \mathrm{CO}$ clumps. However, only one $\mathrm{H}$ II region is identified within the WISE H II catalog, with a slightly lower LSR velocity of $\sim 38 \mathrm{~km} \mathrm{~s}^{-1}$.

Cloud J: We find that Cloud J has a radius of $R_{A} \simeq 13 \mathrm{pc}$ and a mass of $M_{G, A} \simeq 2.0 \times 10^{4} M_{\odot}$. However, Cloud J was included in the GMF catalog by Ragan et al. (2014) in which they estimate a total cloud length of 79 pc. They estimated a total cloud mass of $6.8 \times 10^{4} M_{\odot}$ (part of this difference results from the larger considered area; part from assuming a smaller $n_{12 \mathrm{CO}} / n_{\mathrm{H} 2}$ ratio of $\left.1.1 \times 10^{-4}\right)$. Our analysis also finds that at the largest extraction radii, Cloud $\mathrm{J}$ is a filamentary cloud, extending $\sim 60 \mathrm{pc}$ in the $\mathrm{CE}$ case. Additionally, the co-added distribution of column density with velocity at all cloud radii indicate that Cloud $\mathrm{J}$ is quite symmetric and well isolated in velocity space. IRDC J, i.e., G053.11+00.05 from Simon et al. (2006a), defined the search region, but Cloud J also includes the IRDC G053.31+00.00 within its $30 \mathrm{pc}$ radius. The $8 \mu \mathrm{m}$ images of the Cloud $\mathrm{J}$ reveal a significant amount of bright dust emission, which Ragan et al. (2014) associated with a foreground high-mass star-forming region at $v_{\mathrm{LSR}} \sim 4 \mathrm{~km} \mathrm{~s}^{-1}$. Our search through the WISE H II database found one radio quiet $\mathrm{H}$ II region at $l=53.09, b=0.12$ with an LSR velocity consistent with Cloud J (Anderson et al. 2014).

\subsubsection{Spatial and Velocity Offsets between IRDCs and GMCs}

The spatial and velocity offsets between the IRDC and GMC center of masses are listed in Table 2 quoted to the nearest resolution element of the GRS position-velocity grid. In general, spatial offsets grow as one considers larger GMC scales. At the $30 \mathrm{pc}$ aperture scale applied to connected extraction, this offset is typically $\sim 2-10 \mathrm{pc}$. All but clouds C and I have this GMC center located outside the Simon et al. (2006a) IRDC boundary.

Velocity offsets are typically relatively small, $\sim 1 \mathrm{~km} / \mathrm{s}$. This suggests that IRDCs have been formed by contraction/compression of structures that were already pre-existing within the GMC. Clouds A, B, C, E, I, J have relatively simple CE CO morphologies in position velocity space. The remaining clouds exhibit more complex, multi-component structures. 


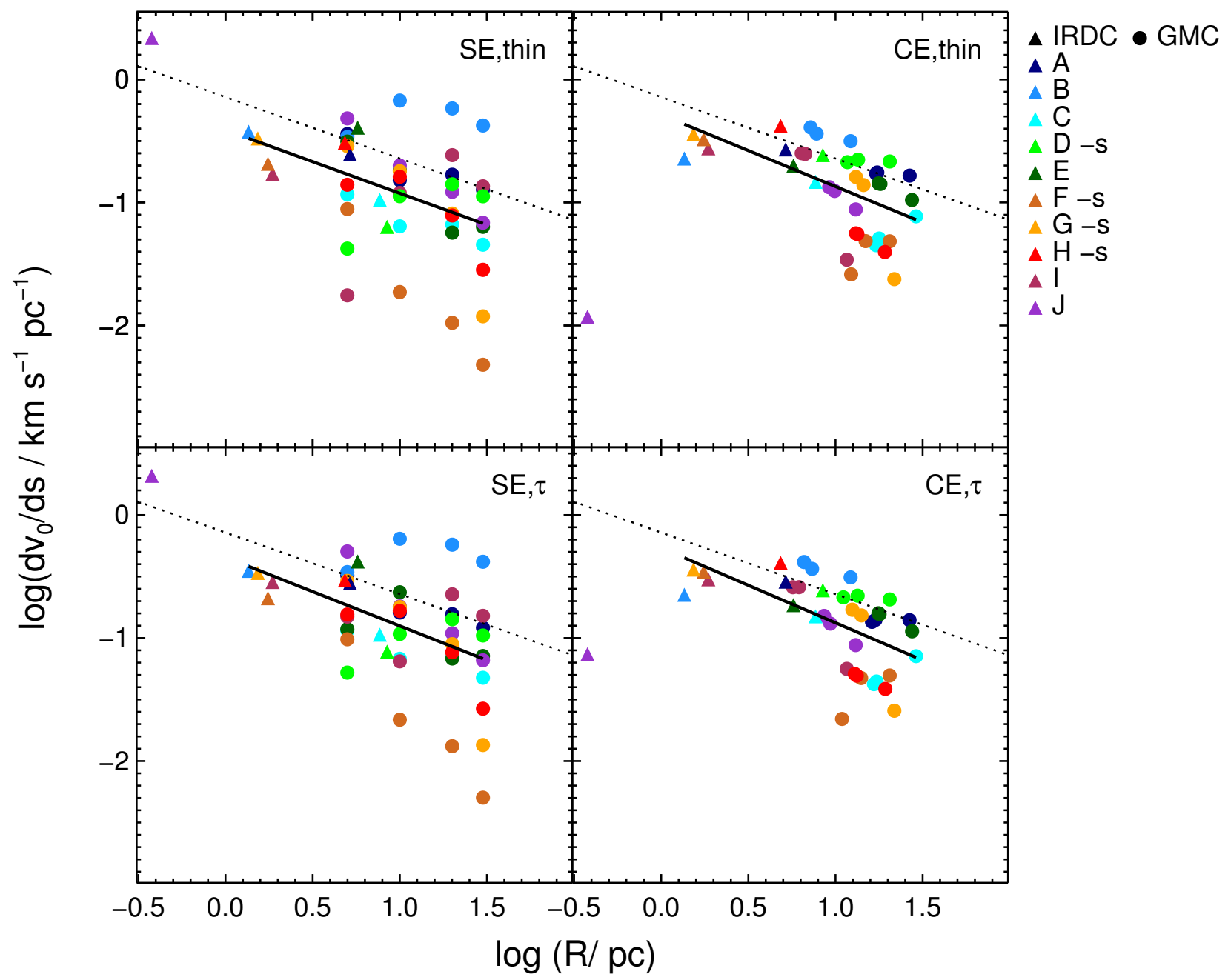

FIG. 5.- Velocity gradient $d v_{0} / d s$ versus radius $R$ of the clouds over which the gradient is evaluated. Each panel represents a different cloud definition: (a) Top left: simple extraction, optically thin column density derivation; (b) Bottom left: simple extraction, opacitycorrected column densities; (c) Top right: connected extraction, optically thin column density derivation; (d) Bottom right: connected extraction, opacity-corrected column densities. $n$ each panel, the 10 IRDCs are shown by the colored filled triangles as defined in the upper right-hand corner. For Clouds D, F, G, and H, these estimates are based on the single component emission profile. GMCs are shown with filled circles. The best-fit power-law relation from McKee \& Ostriker $(2007)\left(d v_{0} / d s \simeq \sigma_{\mathrm{pc}}(s / \mathrm{pc})^{\alpha_{\sigma}-1}\right.$, with values of $\alpha_{\sigma} \simeq 0.5$ and $\sigma_{\mathrm{pc}} \simeq 0.72 \mathrm{~km} \mathrm{~s}^{-1}$ ) is shown by the dotted lines. The global (IRDCs and GMCs, excluding IRDC J) best-fit power-law relation for each panel is shown by the solid lines.

\subsection{Kinematics and Dynamics}

\subsubsection{Velocity Gradients and Implications for Turbulence and Rotation}

The GMCs defined by CE have a mean velocity gradient of $d v_{0, \tau} / d s \simeq 0.14 \mathrm{~km} \mathrm{~s}^{-1} \mathrm{pc}^{-1}$. A similar value of $0.13 \mathrm{~km} \mathrm{~s}^{-1} \mathrm{pc}^{-1}$ was found by Imara \& Blitz (2011) for five GMCs. The $\sim 100$-pc-scale filaments have global velocity gradients that are smaller than these values. Jackson et al. (2010) find $\mathrm{d} v / \mathrm{d} s<0.09 \mathrm{~km} \mathrm{~s}^{-1} \mathrm{pc}^{-1}$ in "Nessie", Battersby \& Bally (2012) find $\mathrm{d} v / \mathrm{d} s<0.05 \mathrm{~km} \mathrm{~s}^{-1} \mathrm{pc}^{-1}$ in their 80-pc long cloud, and Ragan et al. (2014) measure $\mathrm{d} v / \mathrm{d} s \simeq 0.06 \mathrm{~km} \mathrm{~s}^{-1} \mathrm{pc}^{-1}$ as an average of the 7 filaments in their sample.

For the elliptical IRDC regions also defined by CE, we find a mean velocity gradient of $d v_{0 . \tau} / d s \simeq 0.26 \mathrm{~km} \mathrm{~s}^{-1} \mathrm{pc}^{-1}$, larger than that of the GMCs. For comparison, on smaller scales within IRDC H, Henshaw et al. (2014) find global velocity gradients of $0.08,0.07,0.30 \mathrm{~km} \mathrm{~s}^{-1} \mathrm{pc}^{-1}$ in several sub-filaments measured over $\sim 2$ parsec length scales, based on centroid velocities of the dense gas tracer $\mathrm{N}_{2} \mathrm{H}^{+}(1-0)$. Larger local gradients $\sim 1.5-2.5 \mathrm{~km} \mathrm{~s}^{-1} \mathrm{pc}^{-1} \mathrm{can} \mathrm{be}$ present on sub-parsec scales. Ragan et al. (2012) found velocity gradients of 2.4 and $2.1 \mathrm{kms}^{-1} \mathrm{pc}^{-1} \mathrm{within} \mathrm{sub}-\mathrm{pc}$ regions of IRDCs G5.85-0.23 and G24.05-0.22, based on observations of $\mathrm{NH}_{3}(1,1)$.

Observed velocity gradients may be a signature of turbulence (e.g., Burkert \& Bodenheimer 2000, McKee \& Ostriker 2007). For a turbulent line-width-size (LWS) relation of the form $\sigma=\sigma_{\mathrm{pc}}(s / 1 \mathrm{pc})^{\alpha_{\sigma}}$, where values of $\alpha_{\sigma} \simeq 0.5$ and $\sigma_{\mathrm{pc}} \simeq 0.72 \mathrm{~km} \mathrm{~s}^{-1}$ have been reported (McKee \& Ostriker 2007), we expect velocity gradients measured over some 


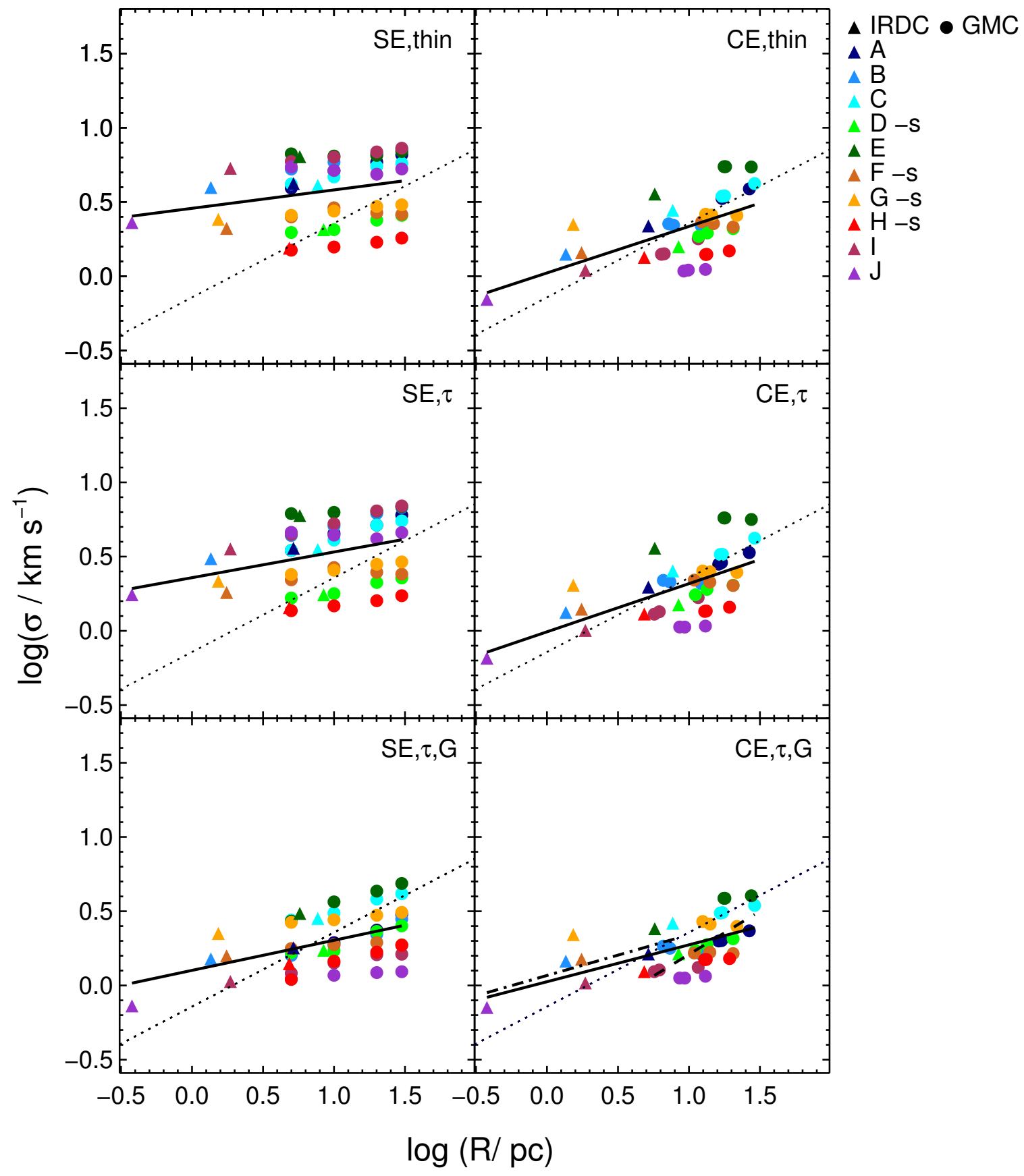

FIG. 6.- Velocity dispersion $\sigma$ versus radius $R$ of the clouds over which the dispersion is evaluated. Each panel represents a different cloud definition: (a) Top left: simple extraction, optically thin column density derivation; (b) Middle left: simple extraction, opacitycorrected column densities; (c) Bottom left: simple extraction, opacity-corrected column densities fitted by a Gaussian profile; (d) Top right: connected extraction, optically thin column density derivation; (e) Middle right: connected extraction, opacity-corrected column densities. (f) Bottom right: connected extraction, opacity-corrected column densities fitted by a Gaussian profile. In each panel, the 10 IRDCs are shown by the colored filled triangles as defined in the upper right-hand corner. For Clouds D, F, G, and H, these estimates are based on the single component emission profile. GMCs are shown with filled circles. The best-fit power-law relation from McKee \& Ostriker (2007) $\left(\sigma=\sigma_{\mathrm{pc}}(\mathrm{s} / 1 \mathrm{pc})^{\alpha_{\sigma}}\right.$, with values of $\alpha_{\sigma} \simeq 0.5$ and $\left.\sigma_{\mathrm{pc}} \simeq 0.72 \mathrm{~km} \mathrm{~s}^{-1}\right)$ is shown by the dotted lines. The global (IRDCs and GMC's) best-hit power-law relation for each panel is shown by the solid lines. In panel $(f)$, the best-fit power-law relations are also shown for the IRDCs (dot-dashed line) and the GMCs (dashed line). 
scale $s$ to vary as $d v_{0} / d s \simeq \sigma_{\mathrm{pc}}(s / \mathrm{pc})^{\alpha_{\sigma}-1}$.

Figure 5 displays our estimates of $d v_{0} / d s$ as a function of cloud size for both IRDCs and GMCs (we note that the small size of IRDC J makes it difficult for velocity gradients to be properly resolved with GRS data, so we exclude it from the following analysis). Fitting the data for clouds defined by $\mathrm{CE}$ and including opacity-corrected column densities (lower-right panel) we find $\alpha_{\sigma}=0.39 \pm 0.14$ and $\sigma_{\mathrm{pc}}=0.54 \pm 0.19 \mathrm{~km} \mathrm{~s}^{-1}$. If each cloud is considered individually, $\alpha_{\sigma}$ has mean and median values of 0.45 and 0.51 , respectively, and a standard deviation of 0.59 . By inspection of the other panels in Fig. 5 we see that the results for the slope and normalization of the power law relation are not too much affected by the choice of cloud definition and mass measurement, but the CE results show smaller dispersions.

We also examine directly the observed velocity dispersions as a function of size scale (effective radius) of the clouds. Figure 6 displays estimates of $\sigma$ as a function of cloud size for both IRDCs and GMCs. Now we see a larger effect on the velocity dispersion versus size relation depending on how the cloud is identified (i.e., $\mathrm{SE}$ or $\mathrm{CE}$ ), how its mass is measured (i.e., whether or not to carry out an opacity correction for the ${ }^{13} \mathrm{CO}$-derived mass), and how to derive velocity dispersion (i.e., via a direct estimate or via a fitted Gaussian). The CE, $\tau$-corrected, Gaussian-fitted results yield a relatively tight power-law, with the smallest dispersion.

Fitting the data for all clouds defined by $\mathrm{CE}$ and including opacity-corrected column densities (lower-right panel) we find $\alpha_{\sigma}=0.25 \pm 0.06$ and $\sigma_{\mathrm{pc}}=1.07 \pm 0.15 \mathrm{~km} \mathrm{~s}^{-1}$. If each cloud is considered individually, $\alpha_{\sigma}$ has a mean and median value of 0.17 and 0.16 , respectively, and a standard deviation of 0.10 . If we fit IRDCs and GMCs separately, we find $\alpha_{\sigma}=0.27 \pm 0.11$ and $\sigma_{\mathrm{pc}}=1.17 \pm 0.16 \mathrm{~km} \mathrm{~s}^{-1}$ for IRDCs and $\alpha_{\sigma}=0.58 \pm 0.13$ and $\sigma_{\mathrm{pc}}=0.43 \pm 0.15 \mathrm{~km} \mathrm{~s}^{-1}$ for GMCs. For virialized, constant mass surface density clouds, where $\sigma \propto(G M / R)^{1 / 2} \propto \Sigma^{1 / 2} R^{1 / 2}($ Larson 1981 McKee \& Ostriker 2007; McKee et al. 2010) one expects $\alpha_{\sigma}=0.5$ and this is consistent with the GMC-only results. However, for IRDCs alone or for the full sample including IRDCs, we see a shallower relation, which could still be consistent with expectations from virial equilibrium since IRDCs have higher mass surface densities. We will discuss the virialization of the clouds in more detail below in 4.3 .3

Observed velocity gradients within molecular clouds can also be interpreted as due to cloud rotation (e.g., Goodman et al. 1993: Imara \& Blitz 2011). With such an interpretation, we assess the projected rotational energy of the clouds $E_{\text {rot }, 0}=(1 / 2) I_{0} \Omega_{0}^{2}$, as discussed in $\S 3.3$. The derived values of $I_{0}$ and $E_{\text {rot. } 0}$ are listed in Table 2 .

We next compare rotational to gravitational energies. Following (Bertoldi \& McKee 1992, hereafter BM92), the gravitational energy of an ellipsoidal cloud is given by

$$
W=\frac{3}{5} a_{1} a_{2} \frac{G M^{2}}{R}
$$

where parameter $a_{1}$ describes the effects of the internal density distribution of the cloud, where for a power-law distribution $\rho \propto r^{-k_{\rho}}, a_{1}=\left(1-k_{\rho} / 3\right) /\left(1-2 k_{\rho} / 5\right)$. Here, for both GMCs and IRDCs, we adopt $k_{\rho}=1$, such that $a_{1}=10 / 9$, based on the IRDC density profile study by BT12. Somewhat steeper density profiles with $k_{\rho} \simeq 1.5$ are derived by BT12 if envelope subtraction is carried out, but this would only change $a_{1}$ by $\sim 10 \%$. The parameter $a_{2}$ accounts for the effect for the cloud's ellipticity. Following BM92 and our ellipsoidal cloud virial analysis from HT11, an ellipsoidal cloud with radius $R$ normal to the axis of symmetry and size $2 Z$ along the axis has an aspect ratio of $y=Z / R$, and semi-major and semi-minor axes of $r_{\max }$ and $r_{\min }$, respectively. Since we do not know the inclination angles of the IRDCs or GMCs, we assume that $R=r_{\min }, Z=r_{\max }$, and that the observed cloud radius is equivalent to the geometric mean radius, $R_{\mathrm{obs}}=\left(R_{\min } R_{\max }\right)^{1 / 2}$. These assumptions differ from those of HT11, where we adopted fiducial inclination angle values of $60^{\circ}$ for the filamentary IRDCs F and $\mathrm{H}$. Now, with a larger sample of both clumpy and filamentary IRDCs, we make no assumption regarding their inclination angles. However, as noted in HT11, an uncertainty of $15^{\circ}$ in inclination would lead to a $\sim 15 \%$ uncertainty in the value of $\mathrm{y}$. We follow the BM92 definition of $a_{2}$, where

$$
a_{2}=\frac{R_{m}}{R} \frac{\operatorname{arcsinh}\left(y^{2}-1\right)^{1 / 2}}{\left(y^{2}-1\right)^{1 / 2}},
$$

where, $R_{m}$ is the mean value of $R_{\text {obs }}$ averaged over all viewing angles. For clouds with low aspect ratios $(y<10)$, BM92 give an approximation for the mean observed radius of $R_{m} / R \sim y^{1 / 2}\left(1 \pm 0.27\left|1-y^{-2}\right|^{0.81}\right)^{0.31}$, accurate to within $2 \%$. Since all of the IRDCs in this study have $y$ values below 10, with IRDC B having the largest aspect ratio of $y=4.67$, the BM92 approximation of $a_{2}$ is valid for use in our gravitational energy estimates.

We thus evaluate $W$, and then the ratio $\beta=E_{\text {rot }} /|W|$, which we also list in Tables 2 and 4 . We find that the GMCs defined by $\mathrm{CE}$ and with opacity-corrected column density estimates have mean $\beta_{\tau} \simeq 0.038$. For the elliptical IRDCs also defined by $\mathrm{CE}$, we find mean $\beta_{\tau} \simeq 0.051$. These values are larger than the $\beta \sim 3 \times 10^{-4}$ values estimated by Ragan et al. (2012) for two IRDCs. On much smaller scales, the study by Goodman et al. (1993) found that most dense cores within dark clouds have values of $\beta \leq 0.18$. The increasing values of $\beta$ that we see on going from GMC to IRDC scales may be indicative of slightly more disturbed kinematics within IRDCs. However, it could also be caused by systematic changes in mass estimation from ${ }^{13} \mathrm{CO}$, e.g., due to increased $\mathrm{CO}$ freeze-out within IRDCs.

\subsection{2. "Rotation" Directions}

Figure 7 displays the distribution of position angles of cloud angular momentum vectors, $\theta_{v, \tau}$, with respect to the direction of Galactic rotation for the cloud definition cases assuming opacity corrected column densities. Our results 


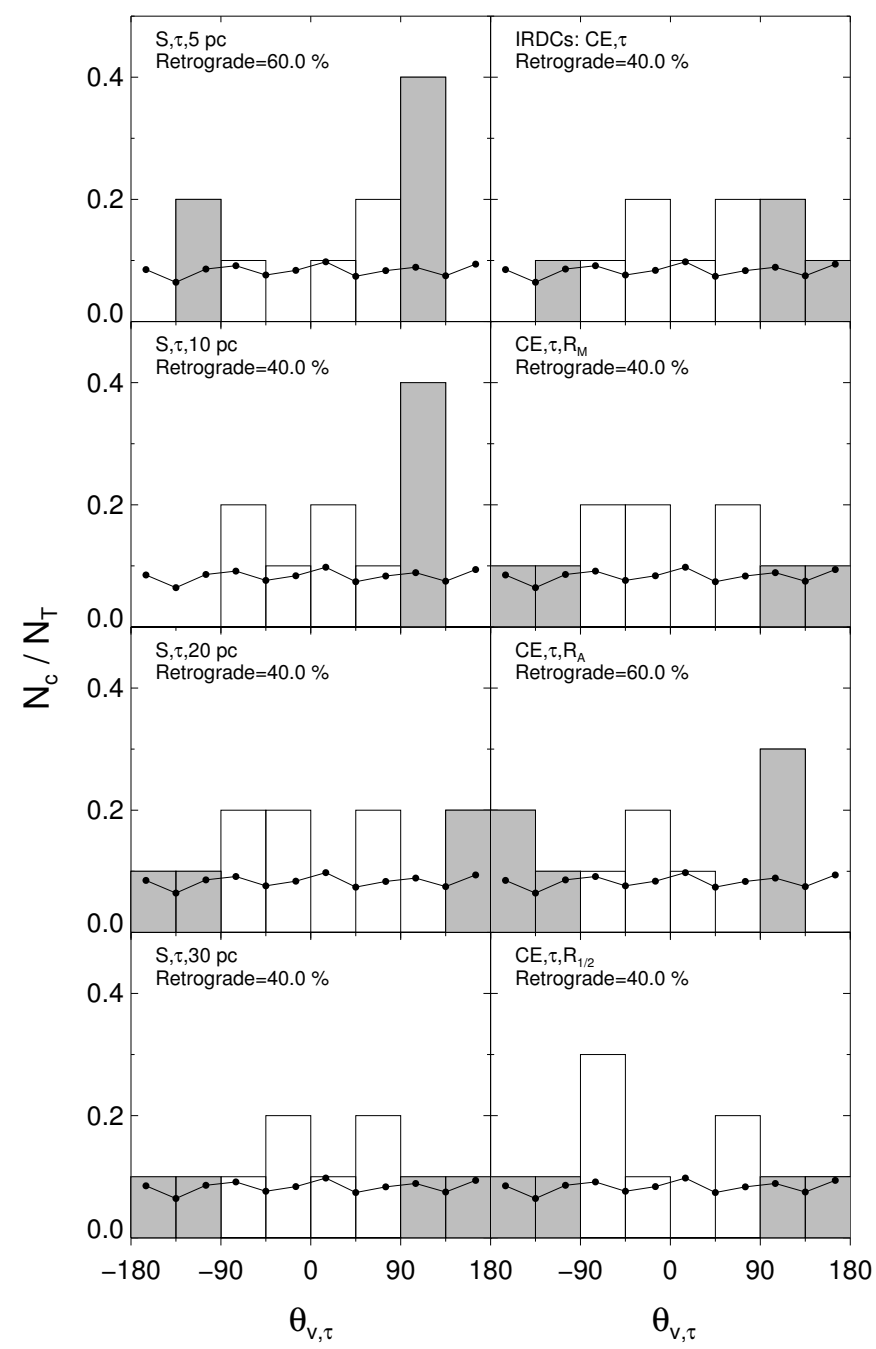

FIG. 7.- Distribution of relative position angle of the angular momentum vectors of the 10 IRDCs/GMCs assuming opacity corrected column densities with respect to the direction of Galactic rotation, $\theta_{v, \tau}$. The effect of different cloud definitions is illustrated in the different panels. In each panel, the black line shows the results from the numerical simulation of Tasker \& Tan (2009) for clouds at $t=250 \mathrm{Myr}$ and the appropriate inclination of viewing angle of $0^{\circ}$ with respect to the galactic plane, i.e., an in-plane view.

show that while a cloud's position angle can vary depending on cloud definition, the overall, global distribution of cloud position angles appears consistent with near equal fractions of clouds with prograde and retrograde rotation, although based on a relatively small sample of only 10 analyzed clouds.

Young GMCs, recently formed from the diffuse ISM should possess position angles which are prograde with Galactic rotation $\left(\theta_{v}<-90^{\circ} ; \theta_{v}>90^{\circ}\right)$. For all of the clouds, measured at various radii for the SE and $\mathrm{CE}$ definitions, we find that there is a large fraction of clouds with retrograde rotation $\left(-90^{\circ}<\theta_{v}<+90^{\circ}\right)$. We find that $\sim 50 \%$ of the $\mathrm{CE}$ clouds analyzed with opacity corrected column densities have retrograde motion.

In the Milky Way, Phillips (1999) compiled a database of rotational measurements for 156 individual clouds from previously published studies, finding that there is a random distribution between the direction of a cloud's angular velocity and the Galactic plane. However, isolated clouds had orientations towards the Galactic poles. They concluded that the rotation of large-scale structures was due to Galactic shear, while the randomized orientation of cloud position angles was due to dynamical and/or magnetic interactions. By estimating the velocity gradients for more than 500 clouds within the GRS, Koda et al. (2006) found that the populations of prograde and retrograde rotation were nearly equal. Beyond the Milky Way, Rosolowsky et al. (2003) found that $40 \%$ of the GMC population in M33 are in retrograde rotation with respect to the galaxy. In a subsequent study of the H I envelopes of 45 GMCs from Rosolowsky et al. (2003), Imara et al. (2011) found that 53\% of these GMCs are in retrograde rotation with respect to the local H I. Furthermore, they suggest that if the linear gradients of the H I envelopes are due to rotation, then $62 \%$ are in retrograde rotation with respect to M33.

In comparison to theoretical models, the disk galaxy simulations of Tasker \& Tan (2009) found near equal amounts of apparent prograde and retrograde clouds $(\sim 50 \%)$ for simulated clouds at $t=250 \mathrm{Myr}$ and a viewing angle of $0^{\circ}$ 
inclination to the galactic plane, i.e., an in-plane view. A substantial fraction of retrograde clouds results if GMCs live long enough to undergo mergers and/or collisions with neighboring clouds. Dobbs \& Pringle (2013) performed 3D smooth particle hydrodynamic (SPH) simulations of GMCs within a galactic disk, including self-gravity and stellar feedback. They found a retrograde GMC population of $\sim 40 \%$, similar to their previous studies (e.g., Dobbs et al. 2011). However, the most massive GMCs tend to be prograde.

Studying the distribution of angular momentum vectors can test scenarios of star formation that involve frequent galactic shear driven cloud-cloud collisions (Tan 2000). At the moment, with only 10 clouds in our pilot sample, we are not yet in a position to make detailed statistical comparison with numerical simulations. We defer such an analysis, using larger samples of Galactic GMCs, to a future paper.

\subsubsection{Cloud Virialization}

Various mechanisms, such as turbulence, magnetic fields, and thermal gas pressure, may be responsible for supporting molecular clouds against gravitational collapse (e.g., McKee \& Ostriker 2007). The dimensionless virial parameter, $\alpha_{\text {vir }}$, describes a cloud's dynamical state via (Bertoldi \& McKee 1992):

$$
\alpha_{\mathrm{vir}} \equiv \frac{5 \sigma^{2} R}{G M}=2 a \frac{E_{K}}{\left|E_{G}\right|}
$$

where $\sigma$ is the 1D mass-averaged velocity dispersion, $a \equiv\left|E_{G}\right| /\left(3 G M^{2} /[5 R]\right)$ is the ratio of gravitational energy, $E_{G}$ (assuming negligible external tides), to that of a uniform sphere, and $E_{K}$ is the kinetic energy. As discussed above in 4.3 for spherical clouds with power-law density distribution, $\rho \propto r^{-k_{\rho}}, a=\left(1-k_{\rho} / 3\right) /\left(1-2 k_{\rho} / 5\right)$, i.e, rising as 1 , $25 / 24,10 / 9,5 / 4,5 / 3$ when $k_{\rho}$ rises as $0,0.5,1,1.5,2$, respectively.

Effects of nonspherical geometry for ellipsoidal clumps have also been considered by BM92, and found to be relatively small ( $\lesssim 10 \%)$ for aspect ratios $Z / R \lesssim 6$, where $Z$ is the clump radius along the axis of symmetry and $R$ the radius normal to this axis. Therefore, here we will ignore these effects of clump elongation.

Ignoring surface pressure and magnetic terms, a cloud in virial equilibrium has $\left|E_{G}\right|=2 E_{K}$, so that $\alpha_{\text {vir }}=a$. Such a cloud in free-fall has $\alpha_{\mathrm{vir}} \rightarrow 2 a$ from below as time progresses. In principle one can imagine a cloud starting with very small levels of internal motion, so that $\alpha_{\mathrm{vir}} \ll 1$, and then crossing through the condition $\alpha_{\mathrm{vir}}=a$ as part of its free-fall collapse. However, such a tranquil and synchronized initial condition would seem to be difficult to achieve in reality if GMCs are accumulating from a turbulent ISM, including potential agglomerating via GMC collisions.

Although it is difficult to differentiate infall motions from virialized turbulent motions, in cases where infall rates have been claimed towards dense clumps, including IRDCs, (e.g., Peretto et al. 2013), the infall velocities are relatively small compared to virial motions (equivalent to the mass doubling times being long $(\sim 10 \times)$ compared to the freefall time; see Tan et al. (2014), for a review). On the larger scales of GMCs, Zuckerman \& Evans (1974) and Krumholz \& Tan (2007) have argued against fast rates of global collapse and infall, since overall star formation rates are much smaller. However, converging flows associated with the collision of independent, self-gravitating clouds may be relatively common (Tan 2000: Tasker \& Tan 2009), and the kinematic signatures of such flows would be difficult to distinguish from global infall.

The effect of surface pressure on the cloud increases the velocity dispersion of the virial equilibrium state and thus raises the value of its virial parameter. For example, for a cloud with $k_{\rho}=1.5$ and negligible surface pressure we have $\alpha_{\mathrm{vir}}=a=5 / 4$. However, if the cloud is embedded in an ambient medium with the cloud's surface pressure equal to the ambient pressure, as in the Turbulent Core model of McKee \& Tan (2003, hereafter MT03), then $\alpha_{\text {vir }}=$ $(5 / 2)\left(3-k_{\rho}\right) /\left[\left(5-2 k_{\rho}\right)\left(k_{\rho}-1\right)\right]=15 / 4$. Note as $k_{\rho} \rightarrow 1$ from above, $\alpha_{\mathrm{vir}} \rightarrow \infty$ (a self-similar singular polytropic sphere with $k_{\rho}=1$ would have a spatially constant pressure, i.e., zero pressure gradient, so there is no self-consistent physical solution; MT03). These results show that virial equilibrium pressure confined singular polytropic spherical clouds with $k_{\rho} \lesssim 1.5$ have virial parameters $\gg 1$.

Support by large-scale magnetic fields acts to reduce the velocity dispersion of the virial equilibrium state. For example, in the fiducial MT03 Turbulent Core model, the mean velocity dispersion of the virial equilibrium state is reduced by a factor $\phi_{B}^{-1 / 2} \rightarrow 2.8^{-1 / 2}=0.598$ if allowing magnetic fields strong enough that the mean Alfvén Mach number is unity. This implies that the value of the virial parameter of the virial equilibrium state would be reduced by a factor of 0.357 .

Magnetic field observations in molecular clouds have been reviewed by Crutcher (2012) and Li et al. (2014). Field strengths increase with density approximately as $B \propto \rho^{2 / 3}$ and are close to values expected for equipartition magnetic and turbulent energy densities and also close (within a factor of $\sim 2$ ) of the critical field strength that would prevent collapse. It thus seems likely that magnetic fields can have significant dynamical effects. Recently, Pillai et al. (2015) have found evidence for strong, dynamically important magnetic fields in two IRDCs, based on relatively ordered directions of polarized sub-millimeter emission, thought to be caused by dust grains that have aligned with the $B$ fields. Unfortunately for the IRDCs/GMCs in our sample, we do not have detailed information on magnetic field strengths.

For the GMCs and IRDCs analyzed in this paper, for each cloud, three virial parameters were estimated. First, we estimated $\alpha_{\text {vir }}$ assuming optically thin conditions to derive $\sigma$ and $M$. Second, using the opacity corrected column densities, $\alpha_{\mathrm{vir}, \tau}$ is estimated using $\sigma_{\tau}$ and $M_{\tau}$. Finally, the virial parameter was estimated using the Gaussian-fitted, opacity corrected column density spectra, i.e., $\alpha_{\mathrm{vir}, \mathrm{G}}$ is estimated using $\sigma_{G}$ and $M_{G}$. In the cases of clouds D, F, G, 


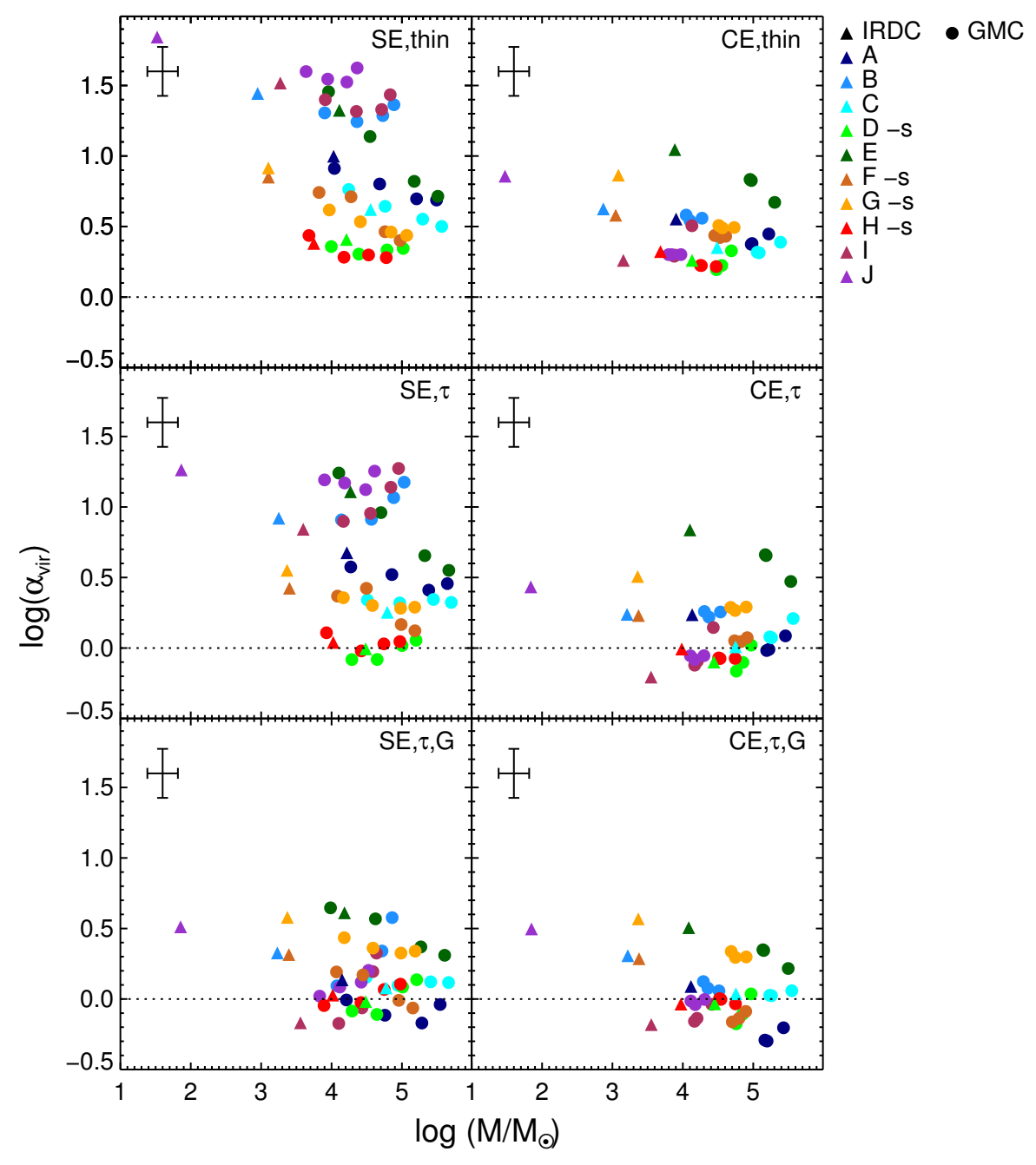

FIG. 8.- Comparison of cloud virial parameter, $\alpha_{\text {vir }}$, with cloud mass. Each panel represents a different cloud definition: (a) Top left: simple extraction, optically thin column density derivation; (b) Middle left: simple extraction, opacity-corrected column densities; (c) Bottom left: simple extraction, opacity-corrected column densities fitted by a Gaussian profile; (d) Top right: connected extraction, optically thin column density derivation; (e) Middle right: connected extraction, opacity-corrected column densities. (f) Bottom right: connected extraction, opacity-corrected column densities fitted by a Gaussian profile. In each panel, the 10 IRDCs are shown by the colored filled triangles (see legend). For Clouds D, F, G, and H, these estimates are based on the isolated single fitted emission profile. The error bar in the top-left of each panel represents the mean uncertainties of $\sim 40 \%$ in $\alpha_{\text {vir }}$ and $\sim 50 \%$ in $M$.

and $\mathrm{H}$, the "-s" mass and velocity dispersions were used to estimate the single component virial parameters of the clouds. If cloud properties are assessed using the full $v_{0} \pm 15 \mathrm{~km} \mathrm{~s}^{-1}$ velocity range (including multiple components), then larger values of $M$ and especially $\sigma$ are inferred, leading to estimates of $\alpha_{\text {vir }}$ that are larger by factors of about 3 . All molecular cloud virial parameters estimates are listed in Tables 2 and 4 . We note that for CE-defined GMCs, the virial analysis is carried out using cloud centers that are the center-of-mass of all the CE gas within the $30 \mathrm{pc}$ radius region. The cloud masses are those that are estimated from the material that is within the radii $R_{M}, R_{A}$ and $R_{1 / 2}$. For SE-defined GMCs, the virial analysis uses the extracted cloud radius as the characteristic size of the cloud.

For the same cloud definition cases, the virial parameter was also estimated for each IRDC. Since these clouds are elliptical, we used the geometric mean observed radius, $R=\sqrt{\left(R_{\operatorname{maj}} R_{\min }\right)}$, as an estimate of the cloud's equivalent spherical shape (but note that, as discussed above, effects of asphericity are minor).

Figure 8 displays the virial parameters as a function of mass for each cloud definition case. The overall range of $\alpha_{\text {vir }}$ is nearly the same for both IRDCs and GMCs. We estimate a typical random uncertainty in $\alpha_{\text {vir }}$ of $\sim 40 \%$ after accounting for uncertainties in the mass surface densities, velocity dispersions, and the cloud kinematic distances. However, systematic uncertainties in mass surface density of a factor of $\sim 2$ would lead to similar levels of uncertainty in $\alpha_{\text {vir }}$. Considering the different cloud definition methods, the virial parameters estimated using the CE are lower than those estimated using SE. For both cloud extraction methods, $\alpha_{\text {vir }}$ is generally smaller for the clouds using opacity corrected column densities compared to those assuming optically thin gas conditions. This trend results from 

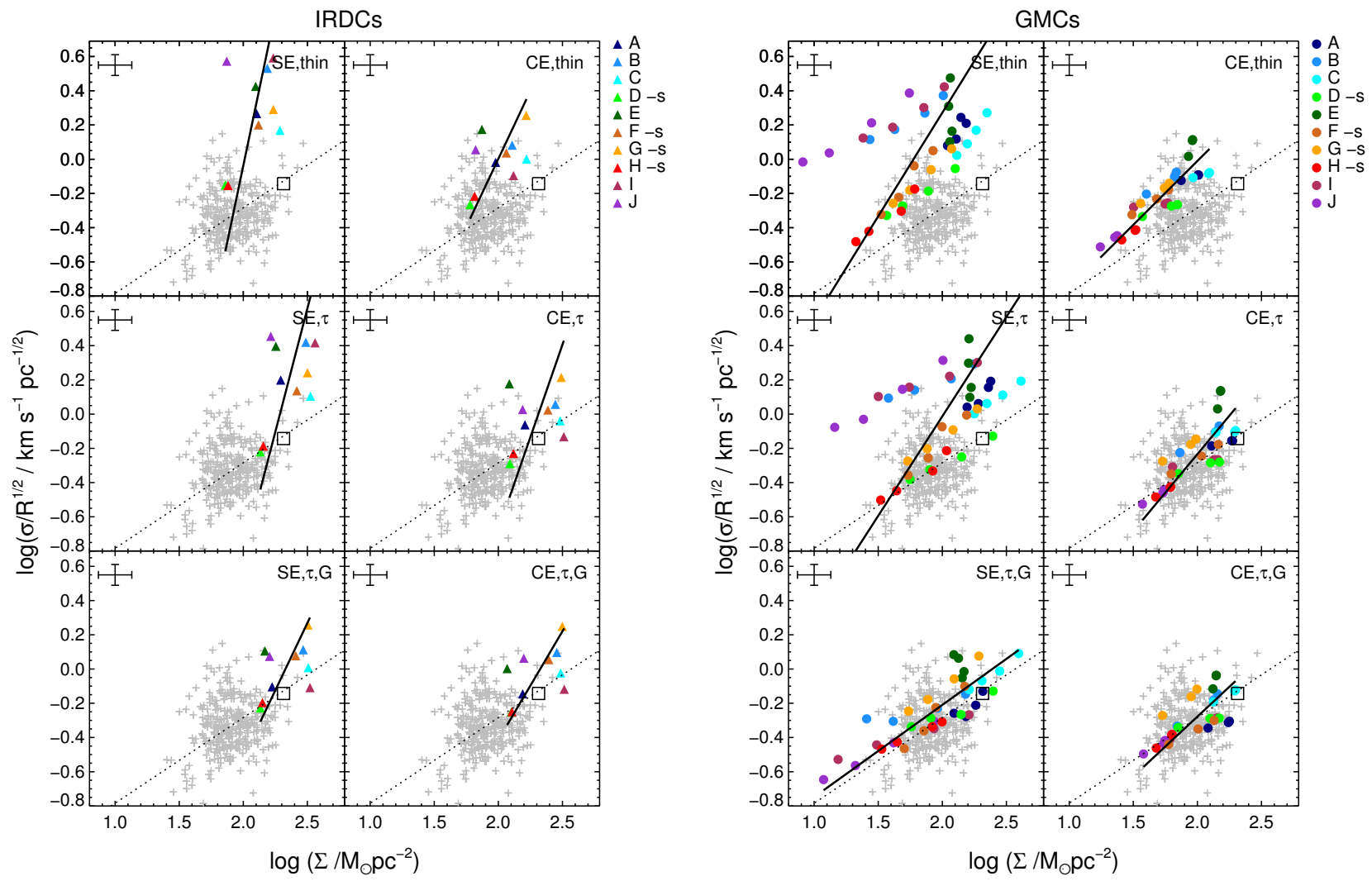

FIG. 9. - Dependence of $\sigma / R^{1 / 2}$ with mass surface density, $\Sigma$. Results for the IRDCs (Left) and GMCs (Right) are shown separately. Each panel represents a different cloud definition: (a) Top left: simple extraction, optically thin column density derivation; (b) Bottom left: simple extraction, opacity-corrected column densities; (c) Middle right: connected extraction, optically thin column density derivation; (d) Middle right: connected extraction, opacity-corrected column densities. (c) Bottom right: connected extraction, optically thin column density, Gaussian fitted profile derivation; (d) Bottom right: connected extraction, opacity-corrected column density, Gaussian fitted profile derivation. In each panel, the IRDCs/GMCs are shown by the colored filled triangles/circles as defined in the upper right-hand corners. For Clouds D, F, G, and H, these estimates are based on the single component emission profiles. The error bar in the upper-left corner represents the mean uncertainties of $\sim 14 \%$ in $\sigma / R^{1 / 2}$ and $\sim 30 \%$ in $\Sigma$. The results of RD10, a total of 329 clouds $\left(M_{>}>10^{4} M_{\odot}\right)$ scaled by a factor of 0.49 to reflect our adopted ${ }^{13} \mathrm{CO}$ abundance (see Tan et al. 2013) are shown by the gray crosses. The dotted line represents virialized conditions with $\alpha_{\text {vir }}=1$, i.e., $\sigma / R^{1 / 2}=(\pi G / 5)^{1 / 2} \Sigma^{1 / 2}$ (see text). The mean results of Solomon et al. (1987) are also shown (black open square). Best-fit power-law relations $\left(\sigma / R^{1 / 2} \propto \Sigma^{n}\right)$ are shown by the solid lines.

larger masses and slightly narrower line profiles in the opacity-corrected cases. Similarly, the smallest $\alpha_{\text {vir values were }}$ estimated in the cases of using the Gaussian fitted spectra to measure both the velocity dispersion and mass, $\sigma_{G}$ and $M_{G}$.

Thus, the virial parameter is sensitive to the method used to extract the ${ }^{13} \mathrm{CO}$ emission associated with a GMC. Overall, the lowest values of $\alpha_{\text {vir }}$ were estimated for the clouds defined by CE, opacity-corrected column densities, and Gaussian fitted masses and velocity dispersions. The mean and median values of these estimates of $\alpha_{\mathrm{vir}, \mathrm{G}}$ are 1.12 and 0.99 , respectively, with a standard deviation of 0.51 (similar to the expected size of the random errors). Similarly, for the IRDCs under the same definitions, the mean and median values of $\alpha_{\mathrm{vir}, \mathrm{G}}$ are 1.88 and 1.93 , respectively, with a standard deviation of 1.11 .

We see that IRDCs have virial parameters that are about a factor of two larger than GMCs. Again, this can be interpreted as evidence for more disturbed kinematics. Alternatively, it could indicate that ${ }^{13} \mathrm{CO}$-derived masses for IRDCs have been underestimated, potentially due to CO depletion.

A population of virialized clouds with similar degrees of pressure confinement may be expected to have a relatively small dispersion in their values of $\alpha_{\mathrm{vir}}$, although some variation is likely due to variation in internal density structure and level of support by large scale magnetic fields. If we denote the mean virial equilibrium value of the virial parameters of such a cloud population as $\bar{\alpha}_{\mathrm{vir}, \text { eq }}$, then from eq. (7) we can derive

$$
\frac{\sigma}{R^{1 / 2}}=\left(\frac{G \pi}{5}\right)^{1 / 2} \alpha_{\text {vir }, \mathrm{eq}}^{1 / 2} \Sigma^{1 / 2}
$$

(see also Heyer et al. 2009, McKee et al. 2010). By examining the observables $\sigma / R^{1 / 2}$ and $\Sigma$ of our IRDC and GMC samples, we can see if they follow this correlation that is expected if the clouds are virialized. We estimate an 


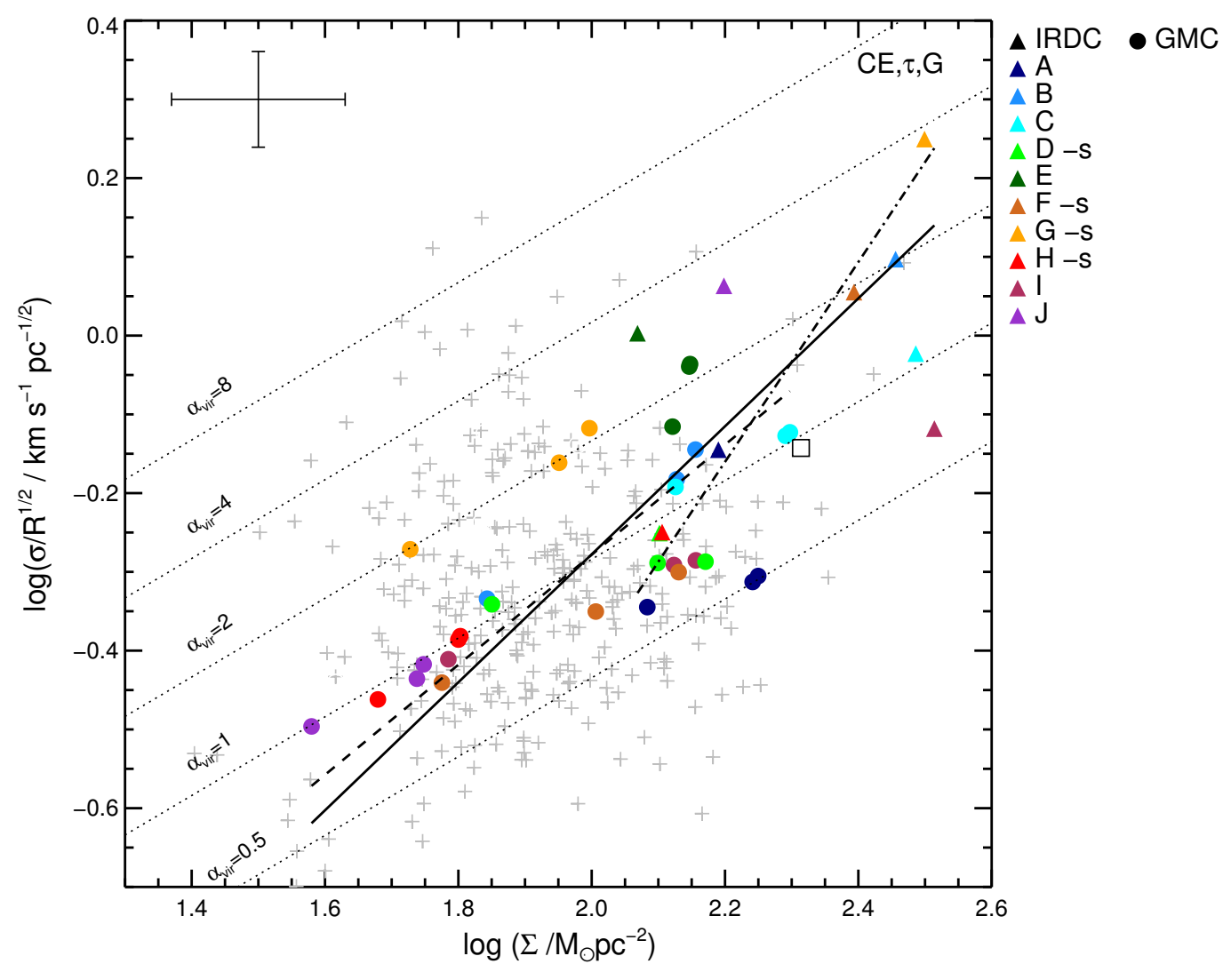

FIG. 10.- Dependence of $\sigma / R^{1 / 2}$ with mass surface density, $\Sigma$, for the all clouds defined by CE, opacity corrected column densities, and gaussian fitted velocity dispersions. As in Figure 9. IRDCs are shown by the filled triangles and the GMCs by the filled circles (see legend). The error bar in the upper-left corner represents the mean uncertainties of $\sim 14 \%$ in $\sigma / R^{1 / 2}$ and $\sim 30 \%$ in $\Sigma$. The results of RD10, a total of 329 clouds with $M>10^{4} M_{\odot}$, are shown by the gray crosses. The mean results of Solomon et al. (1987) are also shown (open black square). The best-fit power-law relation $\left(\sigma / R^{1 / 2} \propto \Sigma^{n}\right)$ is shown for both the IRDCs $(n=1.27 \pm 0.52$; dot-dashed line) and the GMCs $(n=0.70 \pm 0.12$; dashed line). Collectively, the IRDCs and GMCs have a best-fit power-law relation of $n=0.81 \pm 0.10$ (solid line). The dotted lines represents the expected scaling relation for virialized clouds with different values of $\alpha_{\text {vir }}=0.5,1,2,4$, and 8 .

uncertainty in $\sigma / R^{1 / 2}$ of $\sim 14 \%$ based on $10 \%$ uncertainties in both $\sigma$ and $R$.

Thus we considered the above scaling relationship for the results of our ten IRDCs and GMCs (Fig. 9). For IRDCs, $\Sigma$ was estimated for each ellipsoidal cloud using the geometric mean observed radius $R$ and the total ${ }^{13} \mathrm{CO}$-derived mass. For GMCs, $\Sigma$ was estimated using the circular area defined by the extraction radius $R$ and its corresponding ${ }^{13} \mathrm{CO}$-derived mass. We estimate a random uncertainty in $\Sigma$ of $\sim 30 \%$ based on the uncertainty in $T_{\mathrm{ex}}$ and potential variations in our assumed abundances. However, systematic uncertainties could be present at the factor of $\sim 2$ level.

For the ten GMCs, we find that the clouds analyzed using gaussian profile fitting on the opacity corrected column density spectra to estimate the cloud mass and velocity dispersion, yield results most consistent with the scaling expected for gravitational virial equilibrium. Specifically, we find the best-fit power-law relation $\sigma / R^{1 / 2} \propto \Sigma^{n}$ has $n=0.54 \pm 0.05$ and $n=0.70 \pm 0.12$, for the $\mathrm{SE}$ and $\mathrm{CE}$ extraction, respectively. We gauge the significance of these fits by estimating the Pearson's correlation coefficient, $\rho$, and the one-sided $p$-value. For all the GMCs defined by CE and gaussian profile fitting on the opacity corrected column density spectra, we estimate $\rho=0.71$ and a $p$-value of $6.0 \times 10^{-6}$, indicating a significant linearity in the data.

On the other hand, for the ten IRDCs analyzed using gaussian profile fitting on the opacity corrected column density spectra to estimate the cloud mass and velocity dispersion, yield steeper best-fit power-law relations, with $n=1.57 \pm 0.80$ and $n=1.27 \pm 0.52$, for the $\mathrm{SE}$ and $\mathrm{CE}$ extraction, respectively. These power-law fits are less significant than those seen for the GMCs, with $\rho=0.56$ and a $p$-value of 0.047 for the "CE, $\tau, \mathrm{G}$ " method.

Focusing on the "CE, $\tau, \mathrm{G}$ " extraction method, these IRDC and GMC results are displayed together in Fig. 10 Overall the IRDCs and GMCs together yield a best-fit power-law relation of $n=0.81 \pm 0.10$, somewhat steeper than the $n=1 / 2$ expected for virialized clouds. If a power-law relation with $n=0.5$ is fit, then the normalization implies $\bar{\alpha}_{\text {vir }}=1.60$ and 1.03 for IRDCs and GMCs, respectively, and $\bar{\alpha}_{\text {vir }}=1.15$ for the whole sample (with a Pearson's correlation coefficient of $\rho=0.78$ with a $p$-value of $1.7 \times 10^{-9}$ ). We note that some of the dispersion in the data is due to cloud-to-cloud variation, which might be expected if there is systematic variation in degree of surface pressure confinement, magnetization, distance uncertainty (affecting $R$ ) or ${ }^{13} \mathrm{CO}$ abundance. 
We conclude that the relatively tight correlation shown by the data in Fig. 10 is evidence of cloud virialization over a wide range of scales within GMCs, perhaps representing a self-similar hierarchy of self-gravitating virialized structures. There is tentative evidence that IRDCs deviate from this virialized scaling relation in the sense of being more kinematically disturbed. However, the level of deviation of the IRDCs is quite modest and their best-fit $\bar{\alpha}_{\text {vir }} \simeq 1.6$ is a value that is quite compatible with models of virialized singular polytropic $\left(k_{\rho} \simeq 1.5\right)$ spherical clouds embedded in a pressure confining ambient medium. Such a model would yield $\bar{\alpha}_{\text {vir }}=3.75$ (but quite sensitive to $k_{\rho}$ ). Including support from large-scale magnetic fields that lead to Alfvén Mach numbers of unity would lower this to $\bar{\alpha}_{\text {vir }}=1.34$. However, given these uncertainties in $k_{\rho}$, together with those from ${ }^{13} \mathrm{CO}$ abundance, using the absolute value of $\bar{\alpha}_{\text {vir }}$ to assess the degree of virialization is problematic, and we argue it is better to focus on the degree of correlation in the $\sigma / R^{1 / 2}$ versus $\Sigma$ diagram.

For comparison, the results of RD10 for a total of 329 clouds with $M>10^{4} M_{\odot}$ and with their $\Sigma$ values scaled by a factor of 0.49 to reflect our adopted ${ }^{13} \mathrm{CO}$ abundance (see Tan et al. 2013) are also shown in Fig. 10. The RD10 GMCs also follow the expected virialized scaling, but with larger scatter. This is to be expected given that we also see larger scatter with our simpler cloud extraction methods. Consistent with our GMC results, the RD10 GMCs also have values of $\bar{\alpha}_{\text {vir }} \simeq 1.1$.

We also note here that the absolute values of $\Sigma$ that we derive for GMCs are similar to those of H09 and RD10. H09 found an average mass surface density of $\bar{\Sigma} \simeq 42 M_{\odot} \mathrm{pc}^{-2}$ using 162 of the Solomon et al. (1987) 12 CO-defined molecular clouds (but they noted that the expected values are likely underestimated by a factor of $\sim 2$ for these larger scale GMCs due to sub-thermal excitation of $\left.{ }^{13} \mathrm{CO}\right)$. Additionally, the $329 \mathrm{RD} 10$ clouds with $M_{>}>10^{4} M_{\odot}$ yield $\bar{\Sigma} \simeq 88 M_{\odot} \mathrm{pc}^{-2}$. For the 10 GMCs studied here, we find $\bar{\Sigma}_{G} \simeq 80 M_{\odot} \mathrm{pc}^{-2}$ assuming CE, opacity corrected column densities, fitted gaussian line profile to derive mass, and circular cloud radii defined by $R_{A}$. For the 10 IRDCs, defined by the Simon et al. (2006) ellipses, we find $\bar{\Sigma}_{G} \simeq 217 M_{\odot} \mathrm{pc}^{-2}$. However, it is important to note that there is a real spread in $\Sigma$ values between and among the considered IRDC and GMC scales of greater than a factor of 10.

\section{CONCLUSIONS}

We have presented a detailed study of the ${ }^{13} \mathrm{CO}$ gas on various size scales out to radii of $30 \mathrm{pc}$ around 10 well-studied IRDCs (i.e., the sample of BT09 and BT12). We find that all the IRDCs are embedded in a ${ }^{13}$ CO-defined GMC, i.e., a molecular cloud with $M \gtrsim 10^{4} M_{\odot}$. We have taken care to assess the effects of cloud definition, specifically comparing simple extraction of a slab in position-velocity space with extraction only of connected emission features. We have also taken notice of the effects of multiple Gaussian components when they are present. We have considered column density estimates via assuming, first, optically thin ${ }^{13} \mathrm{CO}(1-0)$ emission (with excitation temperatures estimated from associated ${ }^{12} \mathrm{CO}$ emission) and then correcting for line optical depth effects. Our preferred method of "CE, $\tau, \mathrm{G}$ " cloud definition involves connected extraction, opacity-corrected column densities and fitting a single Gaussian profile to derive velocity dispersion and mass.

We discussed the particular morphologies of the individual clouds in both position-velocity space and in relation to plane of sky imaging of the IRDCs. We find most IRDCs are offset from their 30-pc-scale GMC center by $\sim 2-10$ pc, but have only small velocity offsets of $\sim 1 \mathrm{~km} \mathrm{~s}^{-1}$. This suggests IRDCs have been formed by contraction/compression of structures that were already pre-existing with the GMCs rather than being formed from material swept-up by a shock. Six of the ten clouds have relatively simple morphologies in position-velocity space, while the other four exhibit more complex, multi-component structures.

We then focussed on the kinematic and dynamical properties of the clouds. We measured velocity gradients as a function of size scale, $s$, finding they decrease approximately as $s^{-1 / 2}$, i.e., consistent with observations that IRDCs show larger velocity gradients than GMCs. Overall, this is also consistent with the gradients being caused by turbulence with a velocity dispersion versus size relation of the form $\sigma \propto s^{1 / 2}$. Such a scaling is seen in our sample of GMCs, but a somewhat shallower relation is apparent when considering IRDCs and GMCs together. Using velocity gradients to define projected rotation axes we do not see correlations with Galactic plane orientation and have approximately equal fractions of pro- and retrograde rotators with respect to Galactic orbital rotation (though this analysis is limited by the small sample of only 10 clouds). We measure rotational energy to gravitational binding energy ratios of $\beta \simeq 0.045$, with a slight increase seen on going from GMC to IRDC scales.

Assessing virial equilibrium, we find cloud definition has a significant impact on the virial parameter, i.e., the ratio of internal kinetic to gravitational energy. CE, $\tau$,G-defined GMCs have $\bar{\alpha}_{\text {vir }} \simeq 1.0$, while IRDCs have $\bar{\alpha}_{\text {vir }} \simeq 1.6$. We argue that it is difficult to assess the dynamical state, i.e., how close to virial equilibrium, from the absolute values of $\bar{\alpha}_{\text {vir }}$, especially given the uncertain effects of pressure confinement, large-scale magnetic field support and mass surface density estimation from ${ }^{13} \mathrm{CO}$. However, these values $\bar{\alpha}_{\text {vir }} \sim 1.0$ to 1.6 are quite compatible with Turbulent Core/Clump/Cloud models of singular polytropic spherical clouds that are pressure-confined by an ambient medium and have moderate large scale $B$-field support that yields Alfénic Mach numbers of order unity. Note, that ${ }^{13}$ COdefined clouds are likely to have significant surrounding molecular material that could provide pressure confinement.

Rather than focus on absolute values of $\bar{\alpha}_{\text {vir }}$, we follow H09 in examining the predicted scaling in the $\sigma / R^{1 / 2}$ versus $\Sigma$ plane. The results can be sensitive to the method of cloud definition. For our preferred method of CE, $\tau, \mathrm{G}-\mathrm{defined}$ clouds, the GMCs, analyzed over a range of scales, show a strong correlation in this diagram, with a power law slope of $\sigma / R^{1 / 2} \propto \Sigma^{n}$ with $n=0.70 \pm 0.12$, similar to the $n=1 / 2$ value expected under virial equilibrium. Potentially this represents a self-similar hierarchy of self-gravitating virialized structures.

However, the IRDCs exhibit a somewhat steeper scaling with $n=1.27 \pm 0.52$ (or $n=0.81 \pm 0.10$ for the combined 
GMC and IRDC sample). This may indicate that IRDCs have moderately more disturbed kinematics than GMCs, which is qualitative expectation of models in which star formation is triggered by GMC-GMC collisions (e.g., Scoville et al. 1986, $\operatorname{Tan} 2000)$. However, it is also possible that systematic uncertainties affecting mass surface density estimation from ${ }^{13} \mathrm{CO}$, such as increased CO freeze-out in IRDCs (e.g., Hernandez et al. 2011, 2012), are playing a role. Improved methods of measuring mass and assessing degree of CO freeze-out in these clouds are needed to help distinguish these possibilities and thus reliably measure the dynamical state of gas that is likely to form star clusters.

Additional future work will involve applying the techniques developed here to larger samples of clouds to better study connections with Galactic dynamics and Galactic environment, and potential systematic differences of GMCs that are forming IRDCs or that have relatively high dense gas mass fractions.

A.K.H acknowledges support from grants awarded to Bart Wakker at the University of Wisconsin-Madison. We thank Brian Babler for contributing to the GLIMPSE data processing. This publication makes use of molecular line data from the Boston University-FCRAO Galactic Ring Survey (GRS). The GRS is a joint project of Boston University and Five College Radio Astronomy Observatory, funded by the National Science Foundation under grants AST-9800334, AST-0098562, and AST-0100793. J.C.T. acknowledges support from NASA grants ATP09-0094 and ADAP10-0110. 
APPENDIX

A. CLOUD MAPS: B-J

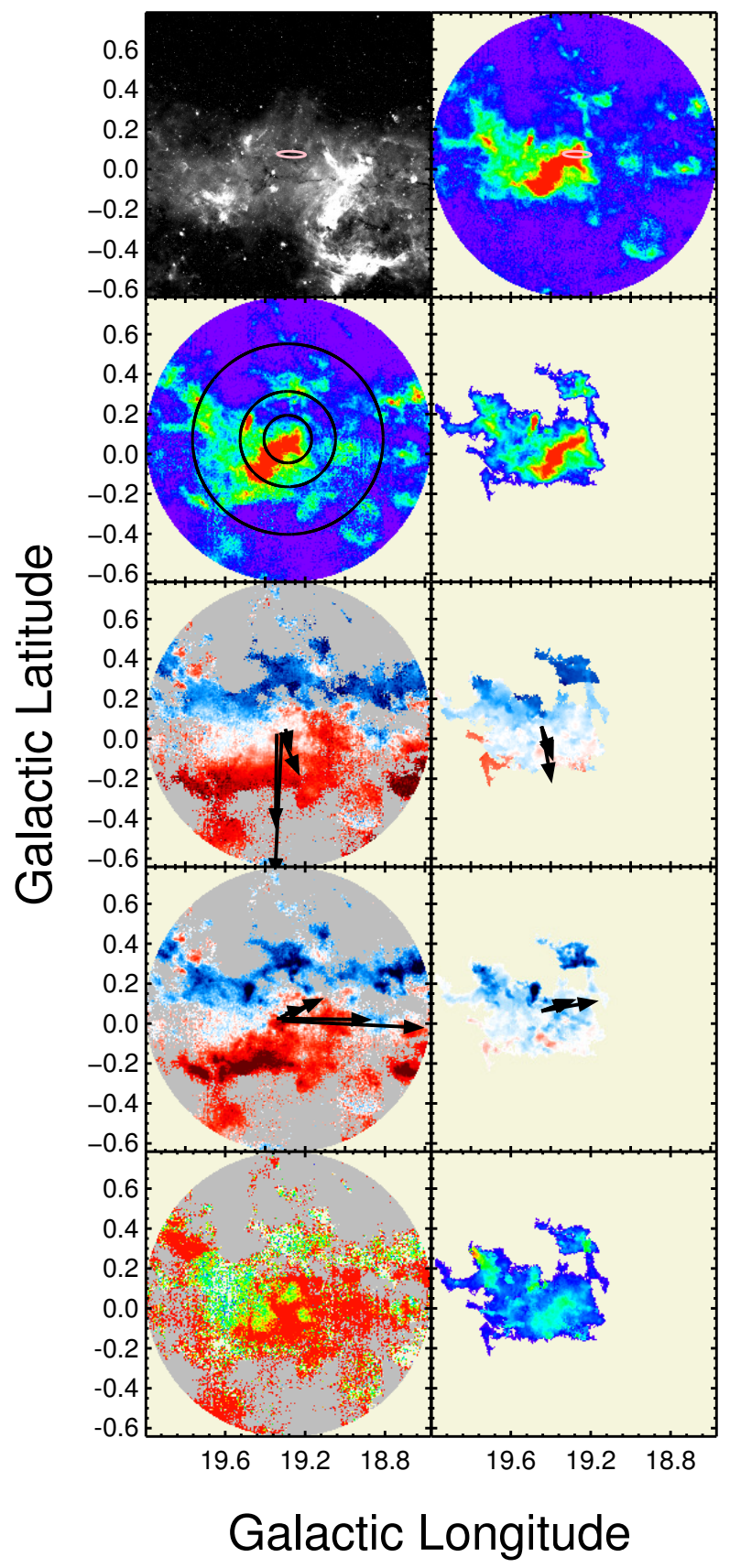

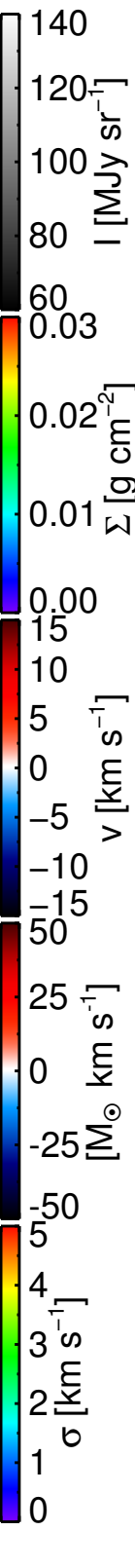

60
0.03

0.02

0.01 Ш

.00

0

$5 \stackrel{5}{z}$

$-10$

$-15$

$25 \%$

$\varepsilon$

$25 \Sigma^{\circ}$

$-50$

$3^{T}$ '

$2 \frac{x}{b}$

1

0
Cloud B

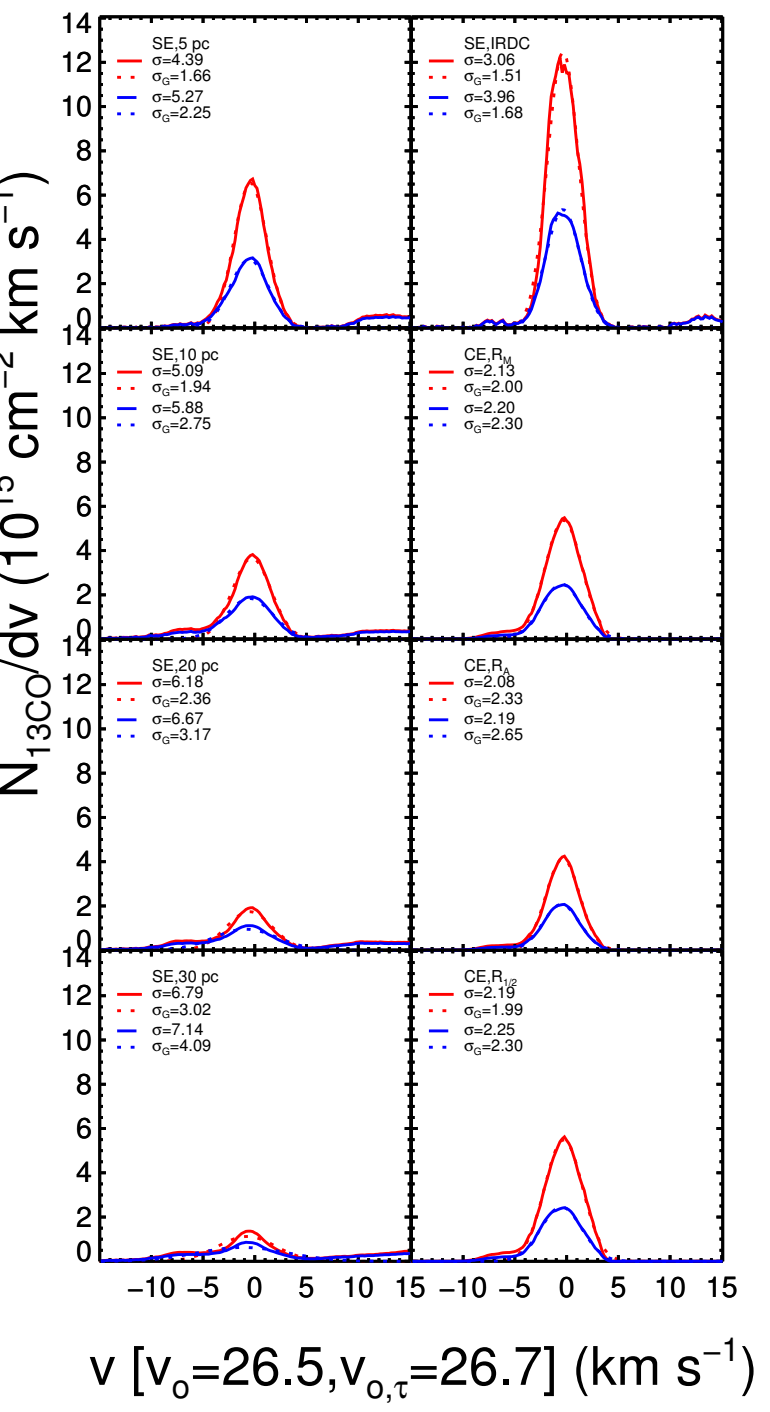

FIG. A1.- Same as Fig. 3 but for Cloud B. The ${ }^{13} \mathrm{CO}(1-0)$ GRS integrated intensity map of the IRDC emission profile is over the velocity range: $v_{\mathrm{LSR}}=20.7-31.6 \mathrm{~km} \mathrm{~s}^{-1}$. 

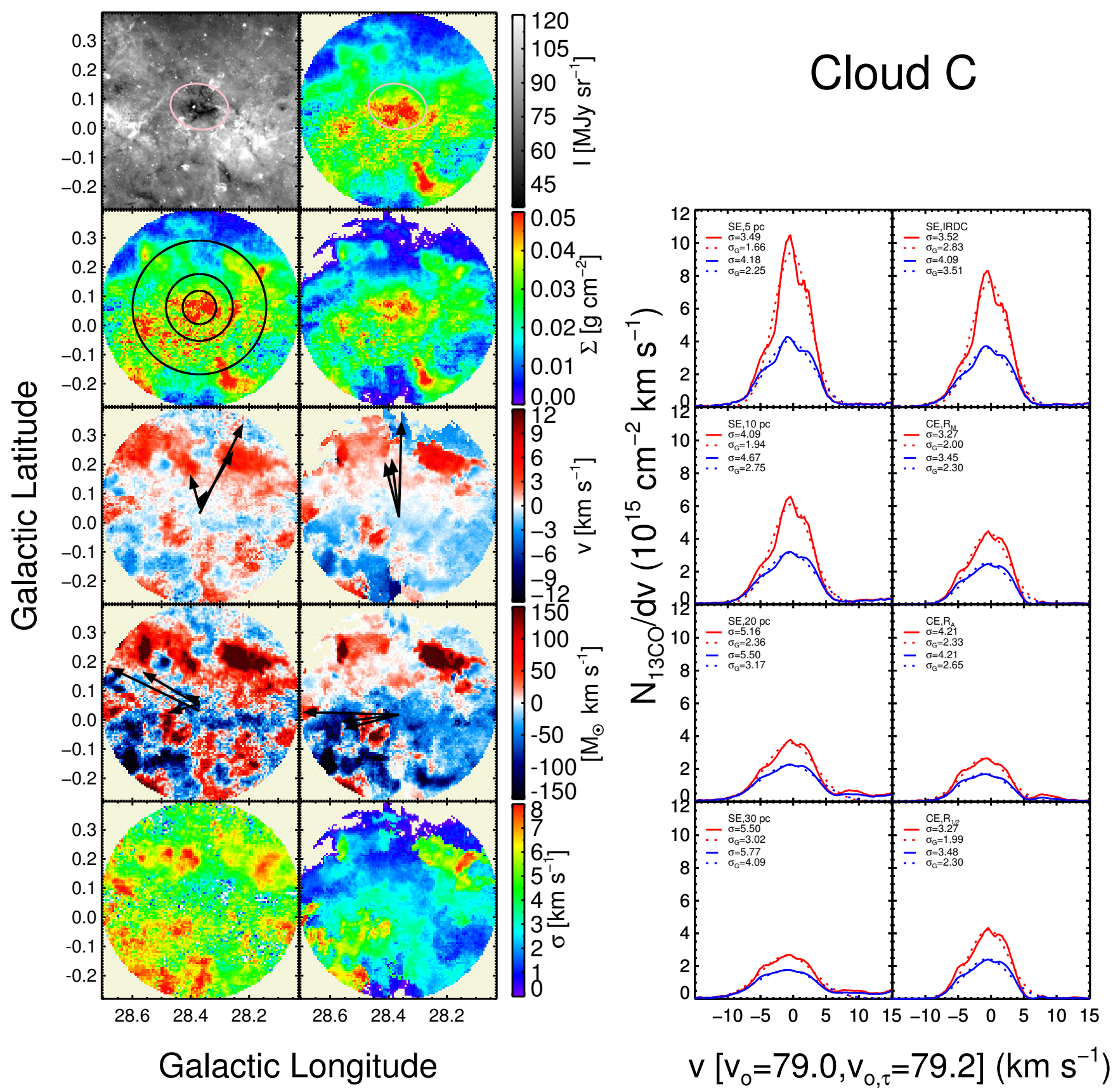

Fig. A2.- Same as Fig. 3 but for Cloud C. The ${ }^{13} \mathrm{CO}(1-0)$ GRS integrated intensity map of the IRDC emission profile is over the velocity range: $v_{\mathrm{LSR}}=66.3-85.7 \mathrm{~km} \mathrm{~s}^{-1}$. 

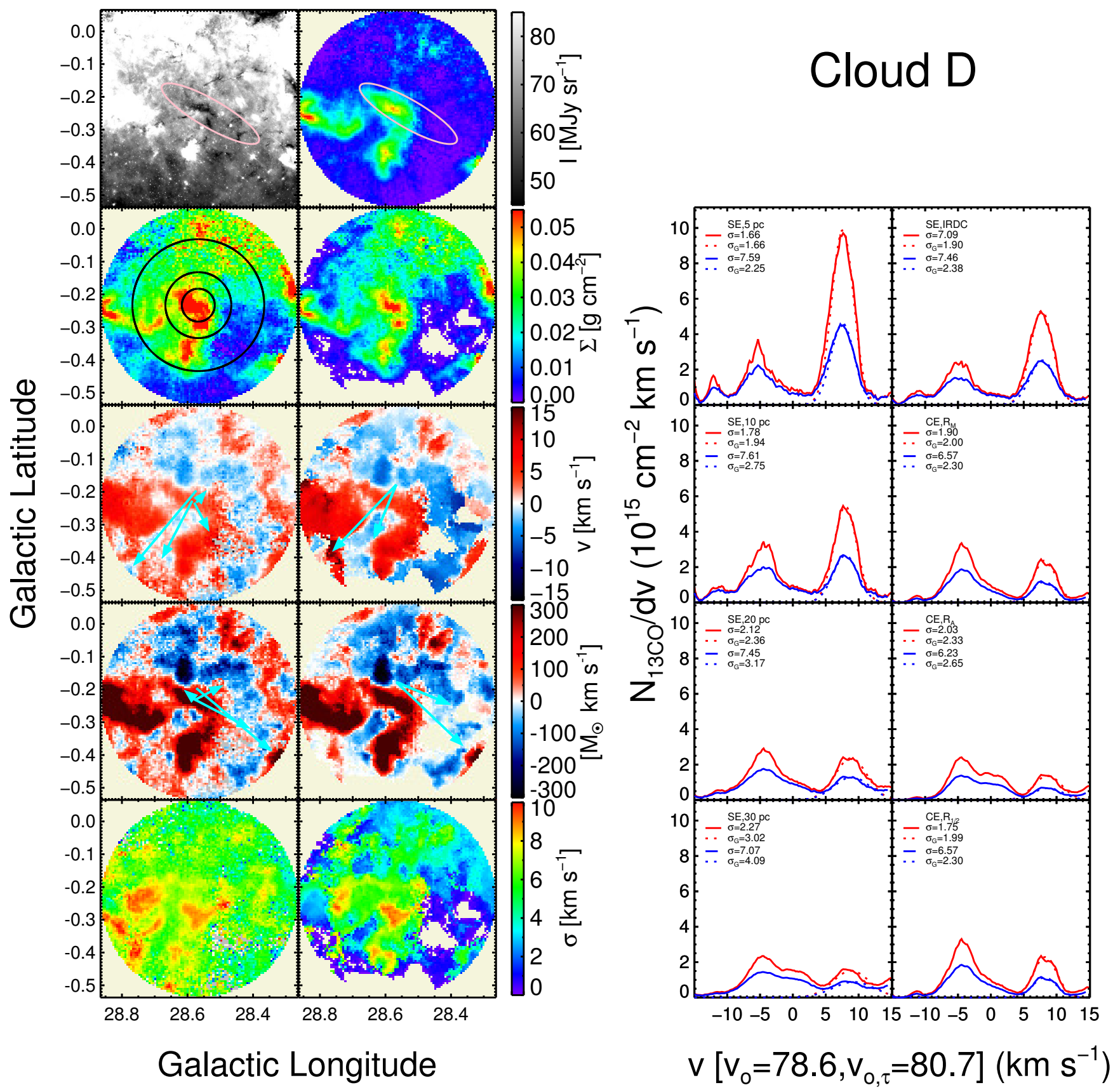

FIG. A3.- Same as Fig. 3 but for Cloud D. The ${ }^{13} \mathrm{CO}(1-0)$ GRS integrated intensity map of the IRDC emission profile is over the velocity range: $v_{\mathrm{LSR}}=84.1-93.7 \mathrm{~km} \mathrm{~s}^{-1}$. 

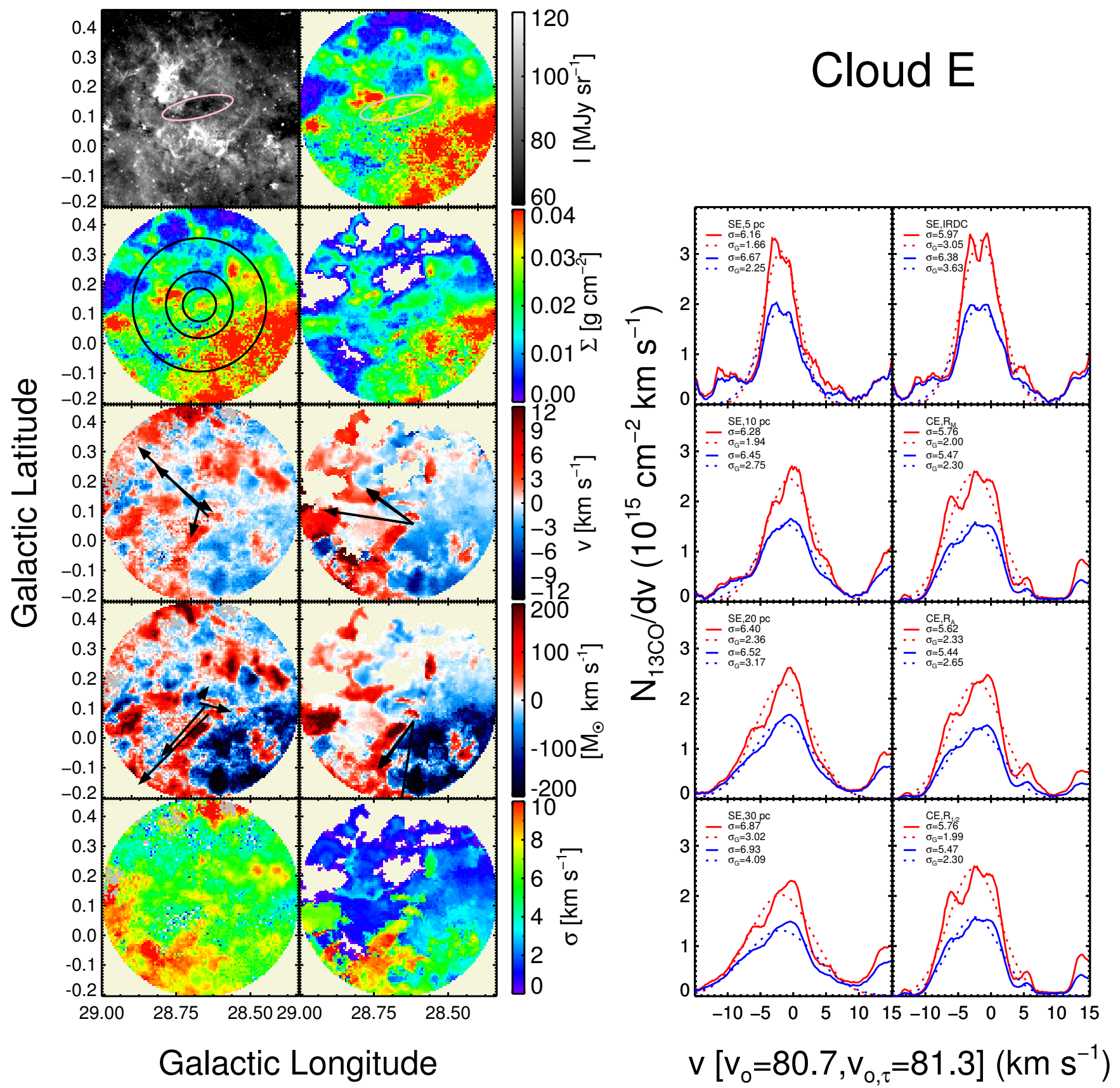

Fig. A4.- Same as Fig. 3, but for Cloud E. The ${ }^{13} \mathrm{CO}(1-0)$ GRS integrated intensity map of the IRDC emission profile is over the velocity range: $v_{\mathrm{LSR}}=66.5-89.8 \mathrm{~km} \mathrm{~s}^{-1}$. 

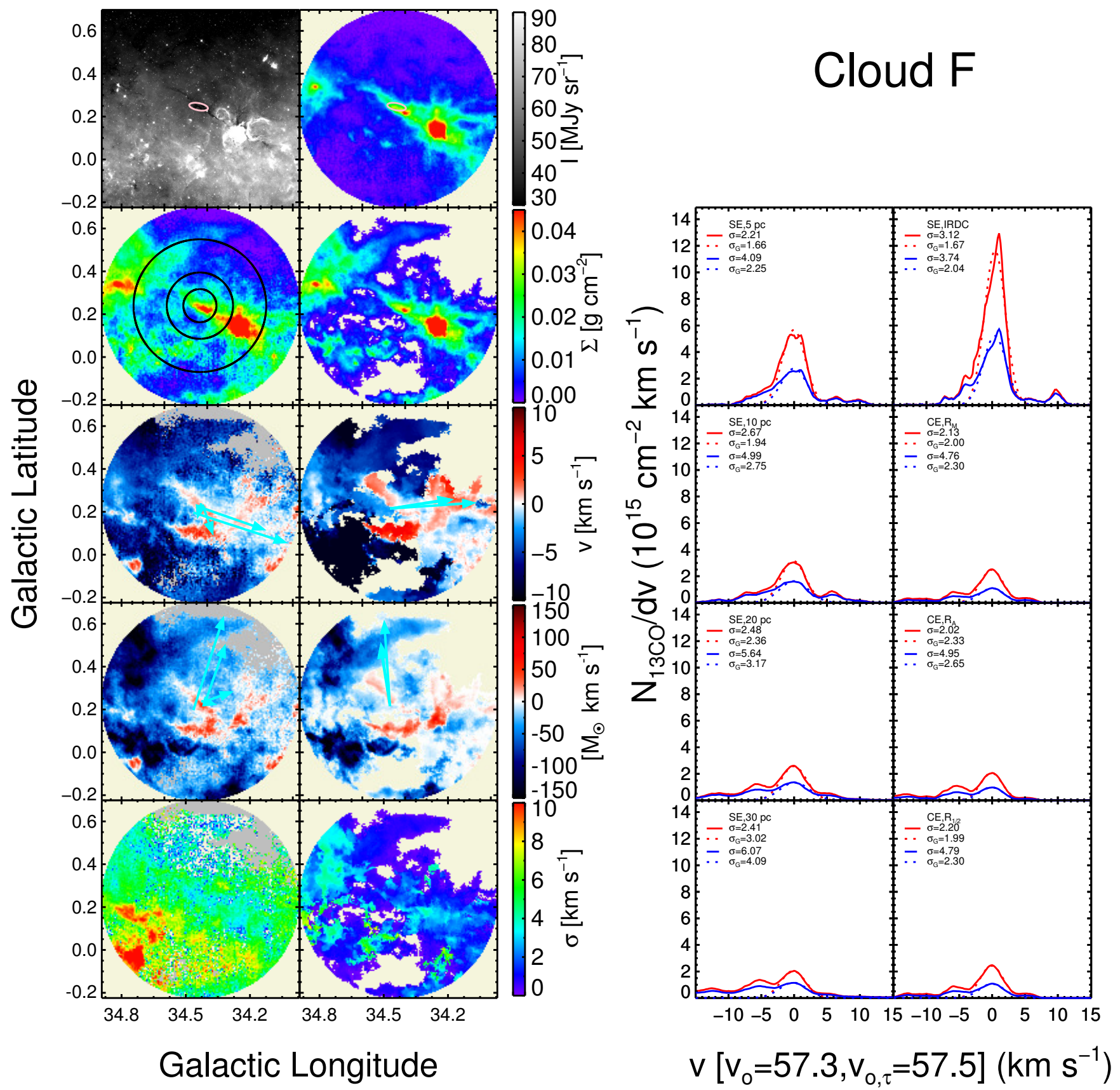

FIG. A5.- Same as Fig. 3 but for Cloud F. The ${ }^{13} \mathrm{CO}(1-0)$ GRS integrated intensity map of the IRDC emission profile is over the velocity range: $v_{\mathrm{LSR}}=54.3-65.6 \mathrm{~km} \mathrm{~s}^{-1}$. 

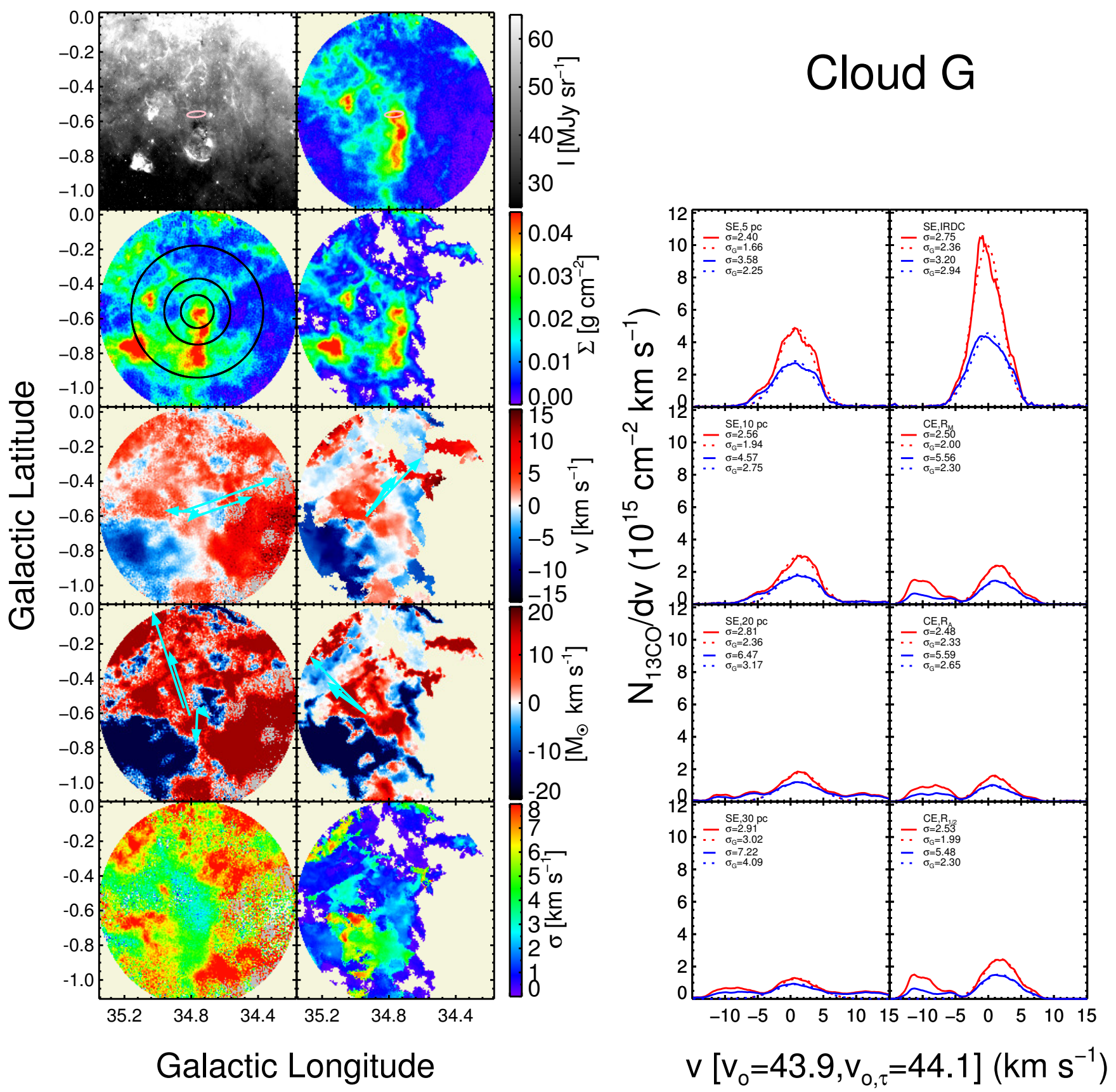

FIG. A6. - Same as Fig. 3 but for Cloud G. The ${ }^{13} \mathrm{CO}(1-0)$ GRS integrated intensity map of the IRDC emission profile is over the velocity range: $v_{\mathrm{LSR}}=39.7-51.8 \mathrm{~km} \mathrm{~s}^{-1}$. 

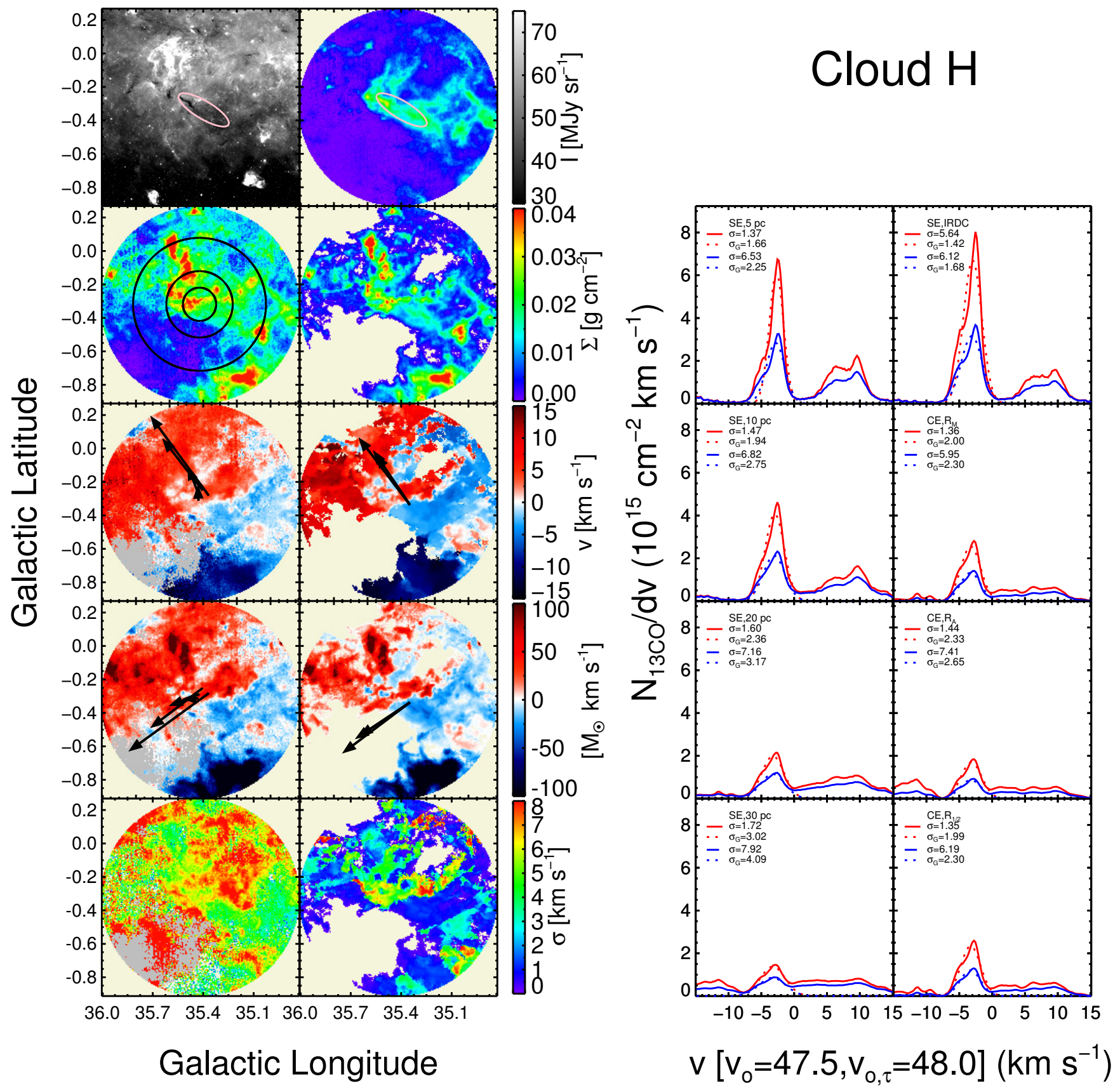

FIG. A7.- Same as Fig. 3 but for Cloud H. The ${ }^{13} \mathrm{CO}(1-0)$ GRS integrated intensity map of the IRDC emission profile is over the velocity range: $v_{\mathrm{LSR}}=39.7-47.1 \mathrm{~km} \mathrm{~s}^{-1}$. 

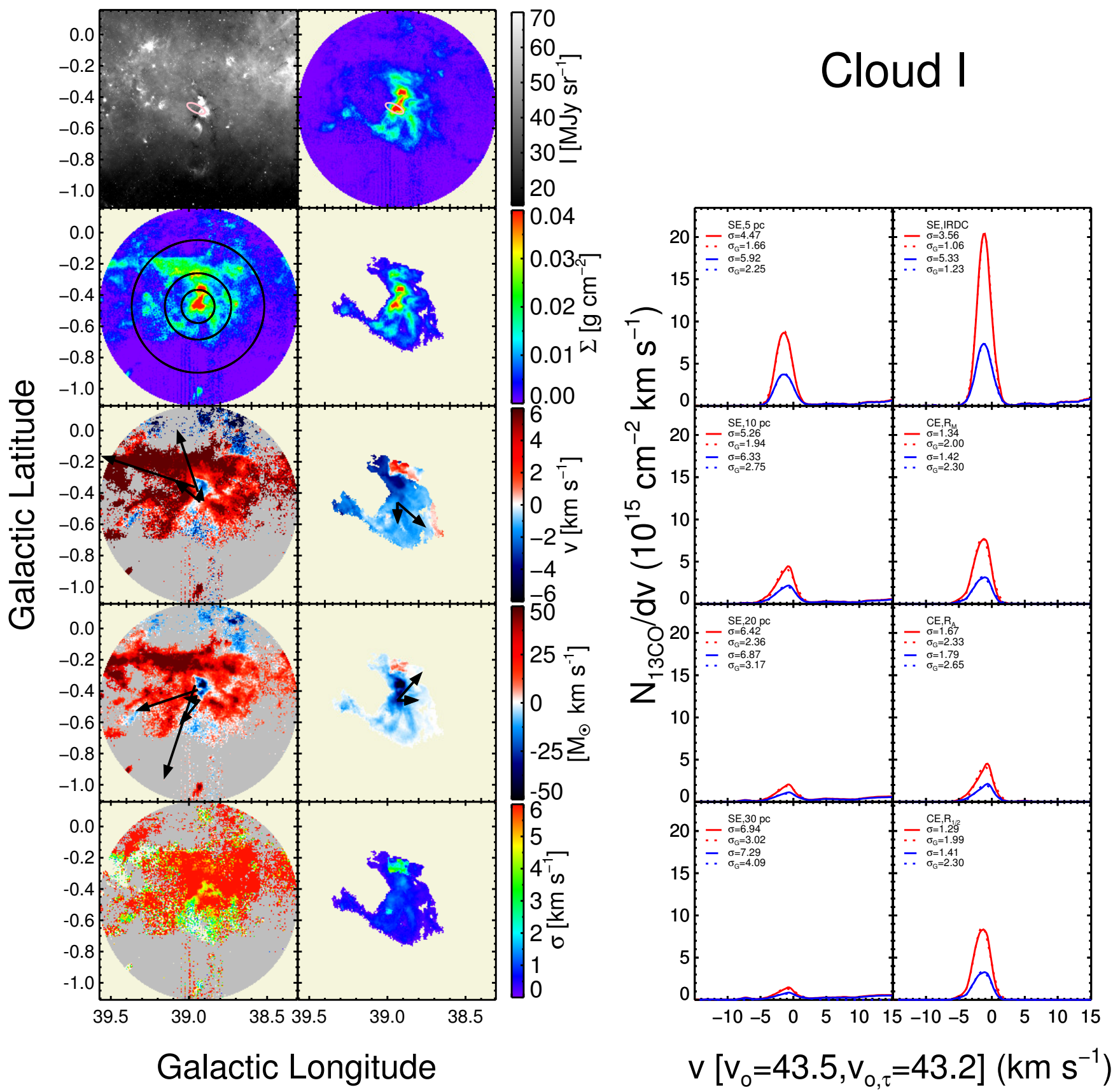

FIG. A8. - Same as Fig. 3 but for Cloud I. The ${ }^{13} \mathrm{CO}(1-0)$ GRS integrated intensity map of the IRDC emission profile is over the velocity range: $v_{\mathrm{LSR}}=37.0-45.1 \mathrm{~km} \mathrm{~s}^{-1}$. 

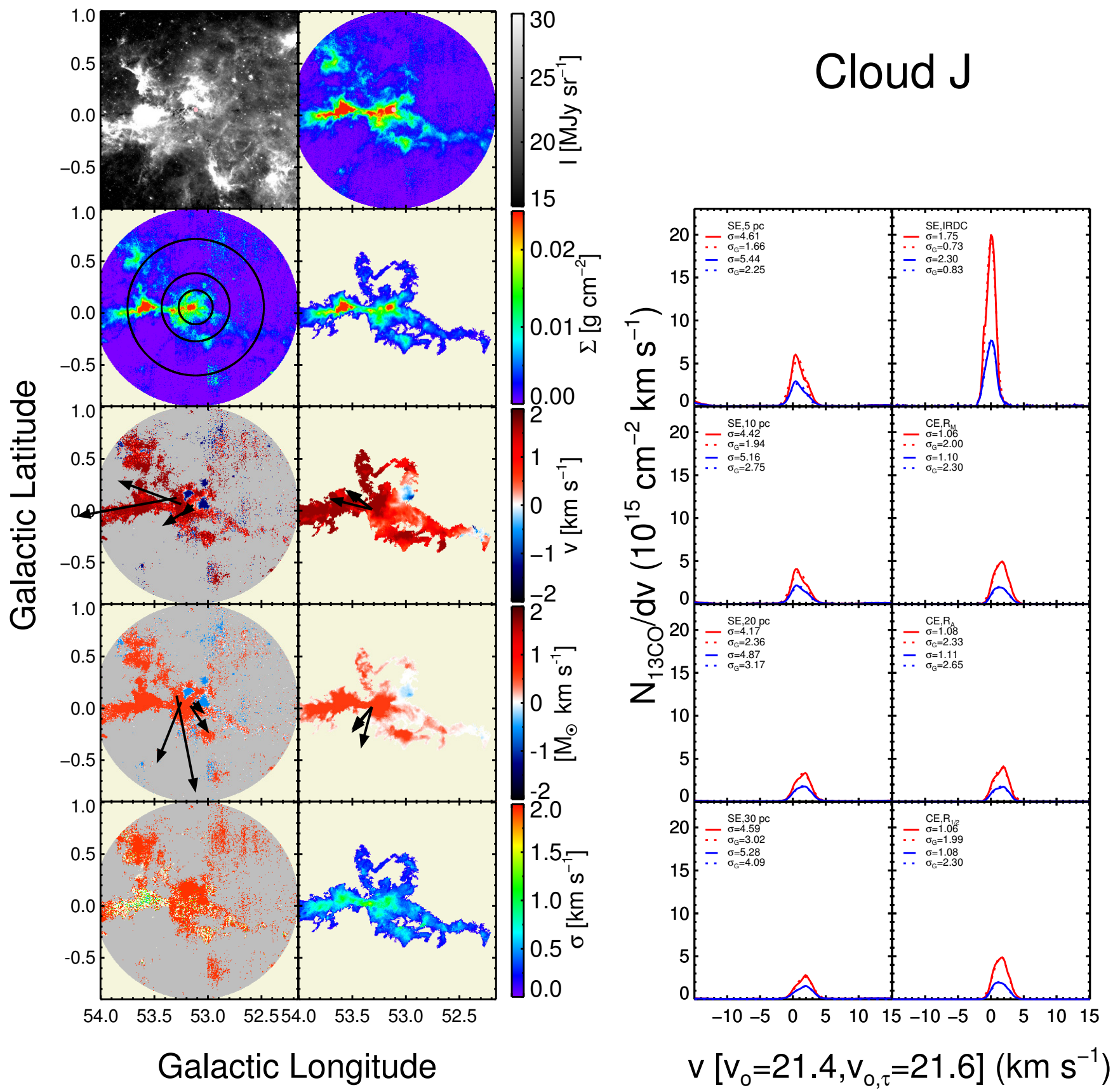

FIG. A9.- Same as Fig. 3 but for Cloud J. The ${ }^{13} \mathrm{CO}(1-0)$ GRS integrated intensity map of the IRDC emission profile is over the velocity range: $v_{\mathrm{LSR}}=18.7-27.1 \mathrm{~km} \mathrm{~s}^{-1}$. 


\section{REFERENCES}

Anderson, L. D., Bania, T. M., Balser, D. S., et al. 2014, ApJS, 212,1

Battersby, C., \& Bally, J. 2012, ArXiv e-prints, arXiv:1208.4608

Battersby, C., Bally, J., Jackson, J. M., et al. 2010, ApJ, 721, 222

Benjamin, R. A., Churchwell, E., Babler, B. L., et al. 2003, PASP, 115,953

Bertoldi, F., \& McKee, C. F. 1992, ApJ, 395, 140

Blitz, L. 1993, in Protostars and Planets III, ed. E. H. Levy \& J. I. Lunine, 125-161

Brunthaler, A., Reid, M. J., Menten, K. M., et al. 2009, ApJ, 693 424

Burkert, A., \& Bodenheimer, P. 2000, ApJ, 543, 822

Butler, M. J., \& Tan, J. C. 2009, ApJ, 696, 484

-. 2012, ApJ, 754, 5

Butler, M. J., Tan, J. C., \& Kainulainen, J. 2014, ApJ, 782, L30

Carey, S. J., Clark, F. O., Egan, M. P., et al. 1998, ApJ, 508, 721

Carey, S. J., Feldman, P. A., Redman, R. O., et al. 2000, ApJ, 543, L157

Chira, R.-A., Beuther, H., Linz, H., et al. 2013, A\&A, 552, A40

Clemens, D. P. 1985, ApJ, 295, 422

Crutcher, R. M. 2012, ARA\&A, 50, 29

Dame, T. M., Hartmann, D., \& Thaddeus, P. 2001, ApJ, 547, 792

Devine, K. 2009, PhD thesis, University of Wisconsin

Dobbs, C. L., Burkert, A., \& Pringle, J. E. 2011, MNRAS, 417, 1318

Dobbs, C. L., \& Pringle, J. E. 2013, MNRAS, 432, 653

Egan, M. P., Shipman, R. F., Price, S. D., et al. 1998, ApJ, 494, L199+

Fontani, F., Caselli, P., Crapsi, A., et al. 2006, A\&A, 460, 709

Foster, J. B., Rosolowsky, E. W., Kauffmann, J., et al. 2009, ApJ, 696, 298

Foster, J. B., Stead, J. J., Benjamin, R. A., Hoare, M. G., \& Jackson, J. M. 2012, ApJ, 751, 157

Goldsmith, P. F., Heyer, M., Narayanan, G., et al. 2008, ApJ, 680,428

Goodman, A. A., Benson, P. J., Fuller, G. A., \& Myers, P. C. 1993, ApJ, 406, 528

Heitsch, F., Slyz, A. D., Devriendt, J. E. G., Hartmann, L. W., \& Burkert, A. 2006, ApJ, 648, 1052

Henshaw, J. D., Caselli, P., Fontani, F., Jiménez-Serra, I., \& Tan, J. C. 2014, MNRAS, 440, 2860

Henshaw, J. D., Caselli, P., Fontani, F., et al. 2013, MNRAS, 428, 3425

Hernandez, A. K., \& Tan, J. C. 2011, ApJ, 730, 44

Hernandez, A. K., Tan, J. C., Caselli, P., et al. 2011, ApJ, 738, 11

Hernandez, A. K., Tan, J. C., Kainulainen, J., et al. 2012, ApJ, 756, L13

Heyer, M., Krawczyk, C., Duval, J., \& Jackson, J. M. 2009, ApJ, 699, 1092

Imara, N., Bigiel, F., \& Blitz, L. 2011, ApJ, 732, 79

Imara, N., \& Blitz, L. 2011, ApJ, 732, 78

Jackson, J. M., Finn, S. C., Chambers, E. T., Rathborne, J. M., \& Simon, R. 2010, ApJ, 719, L185

Jackson, J. M., Rathborne, J. M., Shah, R. Y., et al. 2006, ApJS, 163,145

Jiménez-Serra, I., Caselli, P., Fontani, F., et al. 2014, MNRAS, 439, 1996

Jiménez-Serra, I., Caselli, P., Tan, J. C., et al. 2010, MNRAS, 406, 187

Kainulainen, J., \& Tan, J. C. 2013, A\&A, 549, A53

Kirk, H., Pineda, J. E., Johnstone, D., \& Goodman, A. 2010, ApJ, 723, 457

Koda, J., Sawada, T., Hasegawa, T., \& Scoville, N. Z. 2006, ApJ, 638, 191

Krumholz, M. R., \& McKee, C. F. 2005, ApJ, 630, 250

Krumholz, M. R., \& Tan, J. C. 2007, ApJ, 654, 304

Kurayama, T., Nakagawa, A., Sawada-Satoh, S., et al. 2011, PASJ, 63, 513

Lacy, J. H., Knacke, R., Geballe, T. R., \& Tokunaga, A. T. 1994, ApJ, 428, L69

Lada, C. J., \& Lada, E. A. 2003, ARA\&A, 41, 57

Lada, C. J., Lombardi, M., \& Alves, J. F. 2010, ApJ, 724, 687

Larson, R. B. 1981, MNRAS, 194, 809
Li, H.-B., Goodman, A., Sridharan, T. K., et al. 2014, Protostars and Planets VI, 101

McKee, C. F. 1989, ApJ, 345, 782

McKee, C. F., Li, P. S., \& Klein, R. I. 2010, ApJ, 720, 1612

McKee, C. F., \& Ostriker, E. C. 2007, ARA\&A, 45, 565

McKee, C. F., \& Tan, J. C. 2003, ApJ, 585, 850

Milam, S. N., Savage, C., Brewster, M. A., Ziurys, L. M., \& Wyckoff, S. 2005, ApJ, 634, 1126

Mouschovias, T. 2001, in Astronomical Society of the Pacific Conference Series, Vol. 248, Magnetic Fields Across the Hertzsprung-Russell Diagram, ed. G. Mathys, S. K. Solanki, \& D. T. Wickramasinghe, 515

Nguyen-Lu'o'ng, Q., Motte, F., Carlhoff, P., et al. 2013, ApJ, 775, 88

Padoan, P., \& Nordlund, Å. 2011, ApJ, 730, 40

Perault, M., Omont, A., Simon, G., et al. 1996, A\&A, 315, L165

Peretto, N., \& Fuller, G. A. 2009, A\&A, 505, 405

Peretto, N., Fuller, G. A., Plume, R., et al. 2010, A\&A, 518, L98

Peretto, N., Fuller, G. A., Duarte-Cabral, A., et al. 2013, A\&A, 555, A 112

Phillips, J. P. 1999, A\&AS, 134, 241

Pillai, T., Kauffmann, J., Tan, J. C., et al. 2015, ApJ, 799, 74

Pillai, T., Wyrowski, F., Carey, S. J., \& Menten, K. M. 2006, A\&A, 450, 569

Ragan, S. E., Bergin, E. A., \& Wilner, D. 2011, ApJ, 736, 163

Ragan, S. E., Heitsch, F., Bergin, E. A., \& Wilner, D. 2012, ApJ, 746,174

Ragan, S. E., Henning, T., Tackenberg, J., et al. 2014, A\&A, 568, A73

Rathborne, J. M., Jackson, J. M., \& Simon, R. 2006, ApJ, 641, 389

Rathborne, J. M., Johnson, A. M., Jackson, J. M., Shah, R. Y., \& Simon, R. 2009, ApJS, 182, 131

Reid, M. J., Menten, K. M., Brunthaler, A., et al. 2014, ApJ, 783, 130

Ridge, N. A., Di Francesco, J., Kirk, H., et al. 2006, AJ, 131, 2921

Roman-Duval, J., Jackson, J. M., Heyer, M., et al. 2009, ApJ, 699,1153

Roman-Duval, J., Jackson, J. M., Heyer, M., Rathborne, J., \& Simon, R. 2010, ApJ, 723, 492

Rosolowsky, E., Engargiola, G., Plambeck, R., \& Blitz, L. 2003, ApJ, 599, 258

Samal, M. R., Zavagno, A., Deharveng, L., et al. 2014, A\&A, 566, A122

Sanhueza, P., Jackson, J. M., Foster, J. B., et al. 2013, ApJ, 773, 123

Scoville, N. Z., Sanders, D. B., \& Clemens, D. P. 1986, ApJ, 310, L77

Shimajiri, Y., Kawabe, R., Takakuwa, S., et al. 2011, PASJ, 63, 105

Simon, R., Jackson, J. M., Bania, T. M., et al. 2001, in Bulletin of the American Astronomical Society, Vol. 33, American Astronomical Society Meeting Abstracts, 1450-+

Simon, R., Jackson, J. M., Rathborne, J. M., \& Chambers, E. T. 2006a, ApJ, 639, 227

Simon, R., Rathborne, J. M., Shah, R. Y., Jackson, J. M., \& Chambers, E. T. 2006b, ApJ, 653, 1325

Solomon, P. M., Rivolo, A. R., Barrett, J., \& Yahil, A. 1987, ApJ, 319,730

Tan, J. C. 2000, ApJ, 536, 173

Tan, J. C., Beltrán, M. T., Caselli, P., et al. 2014, Protostars and Planets VI, 149

Tan, J. C., Shaske, S. N., \& Van Loo, S. 2013, in IAU Symposium, Vol. 292, IAU Symposium, ed. T. Wong \& J. Ott, 19-28

Tasker, E. J., \& Tan, J. C. 2009, ApJ, 700, 358

Wienen, M., Wyrowski, F., Schuller, F., et al. 2012, A\&A, 544 A146

Williams, J. P., Blitz, L., \& McKee, C. F. 2000, Protostars and Planets IV, 97

Williams, J. P., de Geus, E. J., \& Blitz, L. 1994, ApJ, 428, 693

Zuckerman, B., \& Evans, II, N. J. 1974, ApJ, 192, L149 